\title{
The Growth and Triggering Mechanisms of the PNA: A MJO-PNA Coherence
}

\author{
Masato MORI \\ Graduate School of Environmental Earth Science, Hokkaido University, Sapporo, Japan \\ and \\ Masahiro WATANABE ${ }^{1}$ \\ Faculty of Environmental Earth Science, Hokkaido University, Sapporo, Japan \\ (Manuscript received 28 June 2007, in final form 6 November 2007)
}

\begin{abstract}
A prominent mode of low-frequency variability in the northern extratropical winter known as the Pacific /North American (PNA) teleconnection pattern prevails not only on seasonal but also on intraseasonal timescales. In this study, processes governing the intraseasonal PNA are investigated using daily fields during 1957-2002. The results of the vorticity budget analysis illustrate that the positive phase of the PNA tends to grow by linear processes such as the barotropic energy conversion from the zonally asymmetric climatological flow. For the negative phase of the PNA, nonlinear low-frequency vorticity advection is as important as the linear processes.

Composite life cycle of the PNA shows that at 9 days before the peak a pronounced wave train was observed along the Asian jet stream and it eventually developed to the PNA near the jet exit region. This wave train is found to be excited by divergent winds primarily associated with anomalous convection of the Madden-Julian Oscillation (MJO). Probability density functions of the MJO calculated separately following the polarity of the PNA reveal a phase locking between the PNA and the MJO. When the active (inactive) convection associated with the MJO reaches the Bay of Bengal to the western Pacific, occurrence frequency of the negative (positive) phase of the PNA is the highest. This MJO triggering explains roughly $30 \%$ of the total PNA events, suggesting that, even though the PNA may be inherent to the extratropical atmosphere, a specific tropical forcing is of importance to realize the PNA as dominant mode.
\end{abstract}

\section{Introduction}

A well-known prominent low-frequency variability in the northern extratropical winter is the

Corresponding author: Masato Mori, Division of Ocean and Atmospheric Sciences, Graduate School of Environmental Earth Science, Hokkaido University, Nishi 5, Kita 10, Sapporo, Hokkaido 060-0810, Japan.

E-mail: masato@ees.hokudai.ac.jp (C)2008, Meteorological Society of Japan

1 Present affiliation: Center for Climate System Research, University of Tokyo, Kashiwa, Japan.
Pacific/North American (PNA) teleconnection pattern (e.g., Wallace and Gutzler 1981; Barnston and Livezey 1987), which represents variability not only on seasonal but also on intraseasonal timescales. The monthly or seasonal mean anomaly of the PNA has been extensively investigated in association with the El Niño-Southern Oscillation (ENSO) phenomenon (e.g., Horel and Wallace 1981). On these timescales, the PNA is generally regarded as steady response to the tropical sea surface temperature (SST) anomalies. On the daily basis, however, the PNA can be detected as fluctuation with the persistence longer than synop- 
tic disturbances. Recently, Feldstein (2000, 2002) showed that the PNA anomaly completes its life cycle of growth and decay within about 2 weeks. Our interest is to understand the characteristics and dynamics of this PNA pattern on submonthly timescale, simply referred to as the low-frequency PNA hereinafter. Since the timescale of the lowfrequency PNA is close to the limit of weather predictability, understanding possible trigger and growth mechanisms is important for predictability of the PNA as well as scientific interest.

There are a number of studies which propose possible mechanisms for the growth of lowfrequency anomalies such as the PNA. They may be classified into three different categories: (i) direct growth due to linear dispersion from a source of topography or diabatic heating (e.g., Hoskins and Karoly 1981), (ii) barotropic growth due to the zonally asymmetric climatological flow (e.g., Simmons et al. 1983; Branstator 1990, 1992; Kimoto et al. 2001; Feldstein 2002; Watanabe and Jin 2004), and (iii) the high-frequency transient eddy feedback (e.g., Lau 1988; Nakamura and Wallace 1993; Branstator 1995; Jin et al. 2006a, b). The studies above, with the exception of (i), suggest that the substantial mechanisms of the growth are inherent to the extratropical atmosphere, and therefore it is difficult to understand that the PNA is directly controlled by a specific external forcing.

Nevertheless, both observational and modeling studies indicate that a coherent fluctuation is found between the extratropical low-frequency variability and the tropical convection associated with the Madden-Julian Oscillation (MJO, Madden and Julian 1971) on the intraseasonal timescale (e.g., Liebmann and Hartmann 1984; Lau and Phillips 1986; Ferranti et al. 1990; Knutson and Weickmann 1987; Hsu 1996; Higgins and Mo 1997; Matthews et al. 2004; Kim et al. 2006). A common finding among these studies is that eastward propagation of the MJO accompanies the significant extratropical low-frequency fluctuations and corresponding modulation of the subtropical jet. The extratropical fluctuation resembles the PNA pattern when the tropical convective activities associated with the MJO reach a specific location. However, most of the studies above examine possible relationships between the MJO and the extratropical circulation on intraseasonal timescales from the tropical point of view, so that it is not clear whether a signal of the MJO is detected when the growth mechanisms of the typical lowfrequency PNA is examined. It also implies that the fractional variance of the low-frequency PNA attributable to the MJO activity has not been quantitatively clarified.

In this study, we focus on the mechanisms influencing the time evolution, especially initiation and growth, of both polarities of the low-frequency PNA. One of our aims is to identify relative roles of the different dynamical mechanisms that have been proposed for the growth of the low-frequency variability, such as energy conversion from the climatological mean flow and the transient eddy feedback, for each stage of the PNA life cycle. This will be investigated by means of daily composites of the low-frequency PNA and associated vorticity budget. Of particular interest is to delineate possible triggering mechanisms of the low-frequency PNA and the extent to which the anomalous convective activities associated with the MJO affect the PNA life cycle. While the PNA growth and decay processes have been investigated by Feldstein (2002) with a similar approach, the triggering mechanisms have not been thoroughly examined. We therefore reexamine the life cycle of the PNA and extend his work by carrying out additional diagnostic analyses.

This paper is organized as follows. In Section 2 , data and analysis procedures are described. In Section 3, the time evolution of composite circulation anomalies associated with the low-frequency PNA is presented. The growth mechanisms for the composite PNA pattern are also examined. Triggering mechanisms and relationship between the PNA and the MJO are investigated in Section 4. Section 5 gives summary and discussion.

\section{Data and Model}

\subsection{Data}

In this study, daily averaged quantities derived from the European Centre for Medium-Range Weather Forecasts (ECMWF) 40-year reanalysis (ERA40; Uppala et al. 2005) are used. The variables are the geopotential height (hereafter referred to as $Z$ ), streamfunction $(\psi)$, velocity potential $(\chi)$, zonal wind $(u)$, and relative vorticity $(\zeta)$ in the upper troposphere either at 200, 300, or $500 \mathrm{hPa}$ (abbreviated such as Z500). The horizontal resolution is $2.5^{\circ} \times 2.5^{\circ}$ and the period of coverage extends from 1957 to 2002 for the boreal winter during November and March. Daily climatology is defined with a slowly varying seasonal 
cycle obtained by taking the calendar mean for each day and then applying a 31-day running mean. Daily anomaly is then defined by subtracting the climatology from raw daily data. To extract low- and high-frequency components of anomalies, a 10-day low-pass (Kushnir and Wallace 1989) and 2-8 days band-pass (Murakami 1979) filters have been applied to these anomaly fields, respectively. In addition, 30-60 days band-pass filtered anomaly of the $200 \mathrm{hPa}$ velocity potential ( $\tilde{\chi} 200)$ was calculated to describe the intraseasonal variability in the tropics.

For measures of the tropical convective activity, we use the National Oceanic and Atmospheric Administration (NOAA) interpolated outgoing longwave radiation (OLR) data. The period of coverage extends from 1979 to 2002 for the winter months. The seasonal cycle is removed and 30-60 days band-pass filter is applied as in $\tilde{\chi} 200$.

\subsection{Definition of the PNA}

As discussed in Feldstein (2002), there is no single spatial pattern that can be referred to as the PNA because of slight differences in definition and data. In this study, the PNA pattern is identified by applying an empirical orthogonal function (EOF) analysis to the 10-day low-pass filtered daily $\psi 500$ anomalies for 45 winters. The EOF analysis is performed over the North Pacific region $(120 \mathrm{E}-60 \mathrm{~W}, 20-90 \mathrm{~N})^{2}$. The leading EOF (EOF1) accounts for $18.1 \%$ of the total variance and is significantly separated from the higher EOFs following the North et al.'s (1982) criterion. The spatial structure of the EOF1 is shown in Fig. 1a, which has wave-like four anomaly centers over the subtropical Pacific, central North Pacific, western Canada, and south eastern US. These features are quite similar to the typical PNA pattern (e.g., Wallace and Gutzler 1981; Barnston and Livezey 1987). Besides, the temporal correlation coefficient between monthly averages of the associated principal components (PC1s) and the PNA index for monthly $Z 500$ defined by Wallace and Gutzler (1981) reaches 0.79 which is significant at the $99 \%$ confidence level. Therefore, the low-frequency PNA can be well captured by this EOF1, and the corresponding PC1 is referred to as the PNA in-

2 The PNA pattern is not identified as the first leading mode when the EOF analysis is performed over the entire Northern Hemisphere without any orthogonal rotation. dex in this study.

Although the magnitude over western Canada appears much smaller than that over other three centers in Fig. 1a, this reflects the fact that the magnitude of circulation anomaly is generally underestimated over the high-latitude region in streamfunction field. Actually, a map of low-pass filtered $Z 500$ anomaly regressed upon the PC1 time series is very similar in shape to the PNA pattern identified by Wallace and Gutzler (1981) over the Pacific/North American region (Fig. 1b).

The power spectral analysis and the histogram of duration defined by a period which the PNA index exceeds one standard deviation reveal that the temporal variability of the PNA index is well approximated by a red noise spectrum with the $e$-folding decay time of 7.1 days (figures not shown), which are consistent with Feldstein (2000). These results indicate that in an attempt to identify the growth and maintenance mechanisms of the low-frequency PNA, we should use daily data at least.

By referring to the decay time of the lowfrequency PNA, we define persistent PNA events when the PNA index exceeds one standard deviation and prolongs 5 days or more. Using these criteria, 84 (88) PNA events for the positive (negative) phase are detected, where a positive PNA is indicative of anomalous low over the North Pacific and vice versa for a negative PNA. We have confirmed that the results shown in this study are not sensitive to slight changes in criteria of the PNA events.

Considering nonlinear nature of the lowfrequency variability, it may be important to argue whether the low-frequency PNA can be identified by a single spatial pattern. According to previous weather regime studies, in a phase space spanned by two leading EOFs of the low-frequency anomaly fields, the regimes (defined by the regions of local maxima in probability density distribution) associated with each phase of the PNA are located approximately symmetrical about the origin point of the phase plane (e.g., Kimoto and Ghil 1993a, b). Therefore, it is justified that the single EOF represents both phases of the low-frequency PNA.

\subsection{Composite analysis procedure}

To describe a typical temporal evolution of the PNA, composite analysis is applied to low-pass filtered daily data. In this subsection, the procedure of composite analysis is briefly described. 
(a)

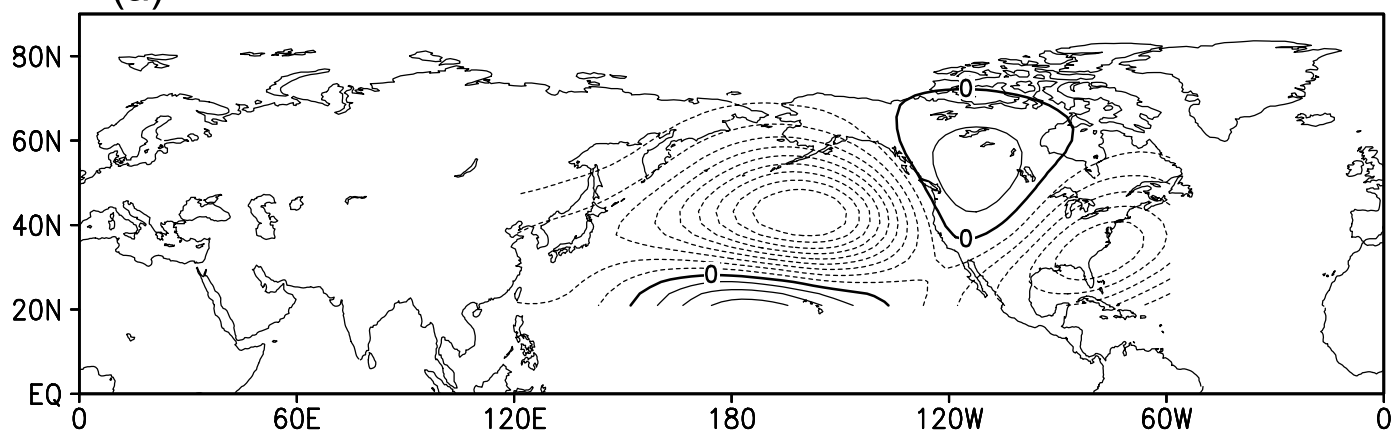

(b)

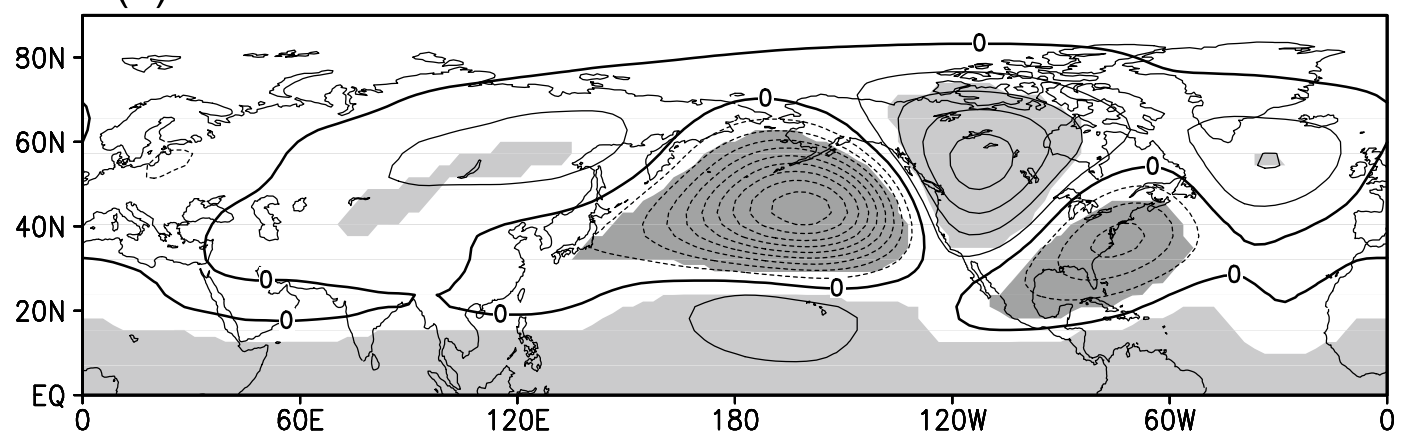

Fig. 1. (a) Spatial structure of EOF1 to 10-day low-pass filtered daily $\psi 500$ anomalies. The contour interval is $1 \times 10^{6} \mathrm{~m}^{2} \mathrm{~s}^{-1}$ and the negative contours are dotted. (b) The 10-day low-pass filtered $Z 500$ anomalies regressed upon the PC1 associated with (a). The contour interval is $10 \mathrm{~m}$. Anomalies significant at the $95 \%$ confidence level are shaded.

Since we are concerned with a typical life cycle of the PNA, relatively strict conditions are used for the composite as follows. For each of 84 positive (88 negative) persistent events, a peak day is first determined by the maximum (minimum) value of the PNA index. Referring to the characteristic time of the PNA, we take 9 days before and after the peak day to make the lagged composite maps. Second, to extract events with monotonous growth and decay, an event is discarded if the PNA indices have peaks more than twice during the analysis period of 19 days. Finally, if the peak of a persistent event occurs within 15 days of the peak of the previous event and both events reveal the same polarity, the latter event is discarded. Following the conditions above, 28 (31) persistent PNA events are retained for the positive (negative) phase. Composite analysis is then performed for each phase.

Time evolution of the PNA event is depicted by composite of the PNA index as shown in Fig. 2. Solid and dashed lines represent positive and negative phases of the PNA event, respectively (Note

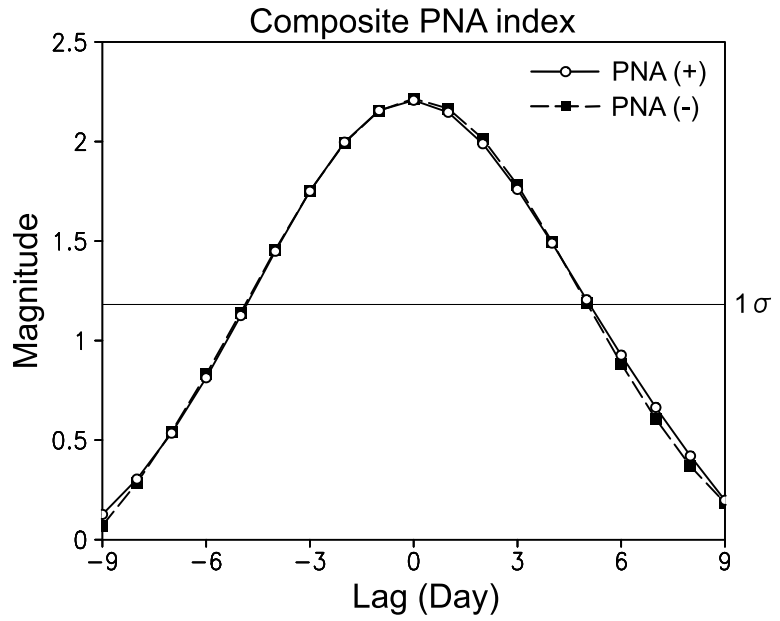

Fig. 2. Composite of the daily PNA index. Solid line with circles (dashed line with squares) represents the positive (negative) phase of the low-frequency PNA. Note the sign reversed for the negative phase. The thin straight line denotes one standard deviation of the PNA index. 
that the sign is reversed for the negative phase). As expected, the composite PNA indices show monotonous growth and decay. Figure 2 also indicates that no significant difference is found between the life cycles of positive and negative PNA events, both completing within approximately 19 days.

\subsection{Budget Analysis}

To identify relative roles of the various dynamical processes in the growth mechanisms for the PNA, vorticity budgets for each life stage of the PNA are examined. The vorticity budget for the low-frequency PNA time evolution is estimated by the streamfunction tendency equation applied to the low-pass filtered $\psi$ (Cai and van den Dool 1994; Feldstein 2002), which can be decomposed as,

$$
\frac{\partial \psi_{L}}{\partial t}=\sum_{i=1}^{7} \xi_{i}+R
$$

where

$$
\begin{aligned}
\xi_{1} & =\nabla^{-2}\left(-\beta v_{L}\right), \\
\xi_{2} & =\nabla^{-2}\left(-[\overline{\boldsymbol{V}}] \cdot \nabla \zeta_{L}-\boldsymbol{V}_{L} \cdot \nabla[\bar{\zeta}]\right), \\
\xi_{3} & =\nabla^{-2}\left(-\overline{\boldsymbol{V}}^{*} \cdot \nabla \zeta_{L}-\boldsymbol{V}_{L} \cdot \nabla \overline{\zeta^{*}}\right), \\
\xi_{4} & =\nabla^{-2}\left\{-(f+\bar{\zeta}) \nabla \cdot \boldsymbol{V}_{\chi L}-\zeta_{L} \nabla \cdot \overline{\boldsymbol{V}_{\chi}}\right\}, \\
\xi_{5} & =\nabla^{-2}\left\{-\nabla \cdot\left(\boldsymbol{V}_{\chi L} \zeta_{L}\right)\right\}_{L}+\nabla^{-2}\left(-\boldsymbol{V}_{\psi L} \cdot \nabla \zeta_{L}\right)_{L}, \\
\xi_{6} & =\nabla^{-2}\left\{-\nabla \cdot\left(\boldsymbol{V}_{\chi H} \zeta_{H}\right)\right\}_{L}+\nabla^{-2}\left(-\boldsymbol{V}_{\psi H} \cdot \nabla \zeta_{H}\right)_{L}, \\
\xi_{7} & =\nabla^{-2}\left\{-\nabla \cdot\left(\boldsymbol{V}_{\chi L} \zeta_{H}\right)\right\}_{L}+\nabla^{-2}\left(-\boldsymbol{V}_{\psi L} \cdot \nabla \zeta_{H}\right)_{L} \\
& +\nabla^{-2}\left\{-\nabla \cdot\left(\boldsymbol{V}_{\chi H} \zeta_{L}\right)\right\}_{L}+\nabla^{-2}\left(-\boldsymbol{V}_{\psi H} \cdot \nabla \zeta_{L}\right)_{L},
\end{aligned}
$$

and $\boldsymbol{V}$ is the horizontal wind vector, $v$ the meridional component of wind, $f$ the Coriolis parameter, and $R$ the residual component such as frictional dissipation and tilting term. For a variable $A, \bar{A}$ denotes the daily climatology of $A,[A]$ the zonal average, and $A^{*}$ the deviation from the zonal average. The subscripts ' $\psi$ ' and ' $\chi$ ' represent the rotational and divergent components of the horizontal wind, respectively, and ' $L$ ' and ' $H$ ' denote the lowand high-frequency components, respectively.

It is important to explain the physical meaning of each term in Eq. (1). The term $\xi_{1}$ is the meridional advection of planetary vorticity by the lowfrequency anomaly, $\xi_{2}\left(\xi_{3}\right)$ the coupling of the anomaly with zonally symmetric (asymmetric) daily climatological flow, $\xi_{4}$ the divergence term, $\xi_{5}$ $\left(\xi_{6}\right)$ the interaction among low- (high-) frequency anomalies, and $\xi_{7}$ their cross-interaction. It is noted that the first four terms, $\xi_{i}(i=1, \ldots, 4)$, represent linear processes while the rest terms, $\xi_{i}(i=5,6,7)$, are nonlinear in terms either of the low-frequency anomaly or of the high-frequency transients.

We have also performed the budget analysis based on the quasi-geostrophic potential vorticity equation and obtained qualitatively similar results to those presented in Section 3.2. However, the results are found to be sensitive to slight changes in setting the boundary condition, so that we employ the simpler vorticity equation.

\subsection{Model}

In an attempt to examine an extratropical response to eastward propagating heat sources associated with MJO, linear baroclinic model (LBM) is used. The LBM consists of primitive equations exactly linearized about a basic state (see Watanabe and Kimoto 2000, 2001, for mathematical expressions). The model variables consist of vorticity, divergence, temperature, and logarithm of surface pressure, which are horizontally expanded by spherical harmonics having the resolution of T42 and vertically discretized by a finite difference to $20 \sigma$ levels. The zonally symmetric or asymmetric winter (November to March) climatology derived from ERA40 is adopted as the basic state. The model also includes three dissipation terms: a biharmonic horizontal diffusion with the damping timescale of 0.5 day for the smallest wave, very weak vertical diffusion (damping timescale is 1000 days) to remove a vertical noise arising from finite difference, and the Newtonian damping and Rayleigh friction as represented by a linear drag, which has the timescale of 0.5 day applied only to the lower boundary layers $(\sigma \geq 0.9)$ and the uppermost two levels $(\sigma \leq 0.3)$.

This LBM is solved with a time-dependent, externally imposed heating which imitates the eastward propagating tropical convection associated with the MJO. The forcing anomaly is represented as follows.

$$
F(\lambda, \phi, \sigma, t)=\alpha(\lambda, t) F_{\phi}(\phi) F_{\sigma}(\sigma),
$$

where $F_{\phi}$ and $F_{\sigma}$ represent meridional and vertical distribution of the forcing, respectively, defined as

$$
\begin{aligned}
& F_{\phi}(\phi)= \begin{cases}0, & \text { if }\left(\phi-\phi_{0}\right)^{2} / \delta_{\phi}^{2}>1, \\
1-\left\{\left(\phi-\phi_{0}\right)^{2} / \delta_{\phi}^{2}\right\}^{1 / 2}, & \text { elsewhere, }\end{cases} \\
& F_{\sigma}(\sigma)=\exp \left\{-\delta_{\sigma}\left(\sigma-\sigma_{0}\right)^{2}\right\},
\end{aligned}
$$


and $\phi_{0}$ is the center latitude, $\delta_{\phi}$ the meridional extent (radius), $\sigma_{0}$ the center level, $\delta_{\sigma}$ the dilation parameter. In this study, we set to $\phi_{0}=0^{\circ}, \delta_{\phi}=10^{\circ}$, $\sigma_{0}=0.45$, and $\delta_{\sigma}=20$. The time- and longitudinaldependent magnitude of the heating is given by $\alpha$, which is estimated from the observational OLR anomalies associated with the MJO cycle. Details for estimating this parameter is presented in Section 4.3.

\section{Life cycles of the PNA}

\subsection{Composite time evolution}

Time evolutional characteristics of the PNA life cycle are described in this section. Lagged composite anomalies of the observed low-frequency $Z 300$ for the positive and negative PNA events are shown in Figs. 3 and 4, respectively. The arrows superimposed indicate horizontal components of the wave-activity flux (WAF) defined by Takaya and Nakamura (2001), which is independent of wave phase and parallel to the local group velocity of Rossby waves in a zonally asymmetric climatological flow. Therefore, this flux is used for representing propagation of Rossby wave packets associated with the low-frequency PNA. Since the quasi-geostrophic approximation has been used in deriving the WAF, the flux has been calculated using geostrophic wind and is shown poleward of $7^{\circ}$ $\mathrm{N}$.

At 6 days before the peak day for the positive phase (Fig. 3a), the pattern is characterized by a wave train emanating from northern Europe to the Pacific while four anomaly centers over the central North Pacific and North America have not yet been formed. The WAF associated with these anomalies indicates the south-eastward and northeastward propagation of Rossby wave energy over Eurasia and the central North Pacific, respectively. From the day -6 to the peak day (Figs. 3a-d), anticyclonic and cyclonic anomalies located over the central North Pacific are found to eventually develop to two upstream centers of the PNA pattern, respectively. Simultaneously, by 4 days before the peak (Fig. 3b), anticyclonic and cyclonic anomalies appear over North America and gradually grow into the two downstream PNA centers afterwards (Figs. 3c-d). After the peak day (Figs. 3e -f), the PNA spatial pattern begins to decay with emitting energy to the downstream. Remarkable difference between the two phases of the PNA life cycle (day -6 to +4 ) is not apparent except that the wave energy propagation from Eurasia is more clearly observed in the positive PNA than in the negative one (Figs. 3a and $4 \mathrm{a}$ ).

One of the notable features of the life cycle of the low-frequency PNA as displayed in Figs. 3 and 4 is that the PNA starts growing from the anomaly center located over the exit region of the Asian jet stream rather than over the subtropical Pacific. As mentioned in the introduction, the time-mean extratropical response to SST anomalies associated with ENSO events resembles the PNA pattern, which is characterized by a wave train emanating from the tropics (e.g., Hoskins and Karoly 1981; Karoly et al. 1989). In daily life cycle, however, it is shown that the wave energy with a large amount has been generated in-situ over the midlatitude Pacific. Besides, the wave activity coming out from the subtropical anomaly is weak and the direction is sometimes equatorward. These features suggest that the low-frequency PNA is not induced by the diabatic heating over the tropical central Pacific and the growth of the PNA is caused by the internal dynamics of the midlatitude atmosphere as discussed in previous studies. The growth mechanisms will be examined in the next subsection.

At day -6 , it seems that the circulation anomalies which eventually develop to two upstream centers of the PNA pattern have already been excited over the midlatitude Pacific (Figs. 3a and 4a). Therefore, it is necessary to examine precursors in earlier stage in order to identify the initiation of the PNA, which is discussed in more detail in Section 4.1.

\subsection{Budget analysis}

In order to understand the growth mechanisms of the PNA, vorticity budget for the composite PNA time evolution is examined using Eq. (1). First, all data are interpolated to the T42 Gaussian grid using a spline function. Second, every eight terms $\left(\xi_{1}, \ldots, \xi_{7}\right.$ and $\partial \psi_{L} / \partial t$, neglecting the residual) were calculated individually from 300 $\mathrm{hPa}$ observational data set and then each of these terms was composited for both phases of the PNA following the same procedure as mentioned in Section 2.3. The streamfunction tendency $\partial \psi_{L} / \partial t$ is calculated simply using a centered finite difference. When the right-hand side of Eq. (1) is calculated, the inverse Laplacian is taken by expanding the field to the spherical harmonics. To estimate the extent to which the individual terms can force a typical positive (negative) PNA life cycle, various 


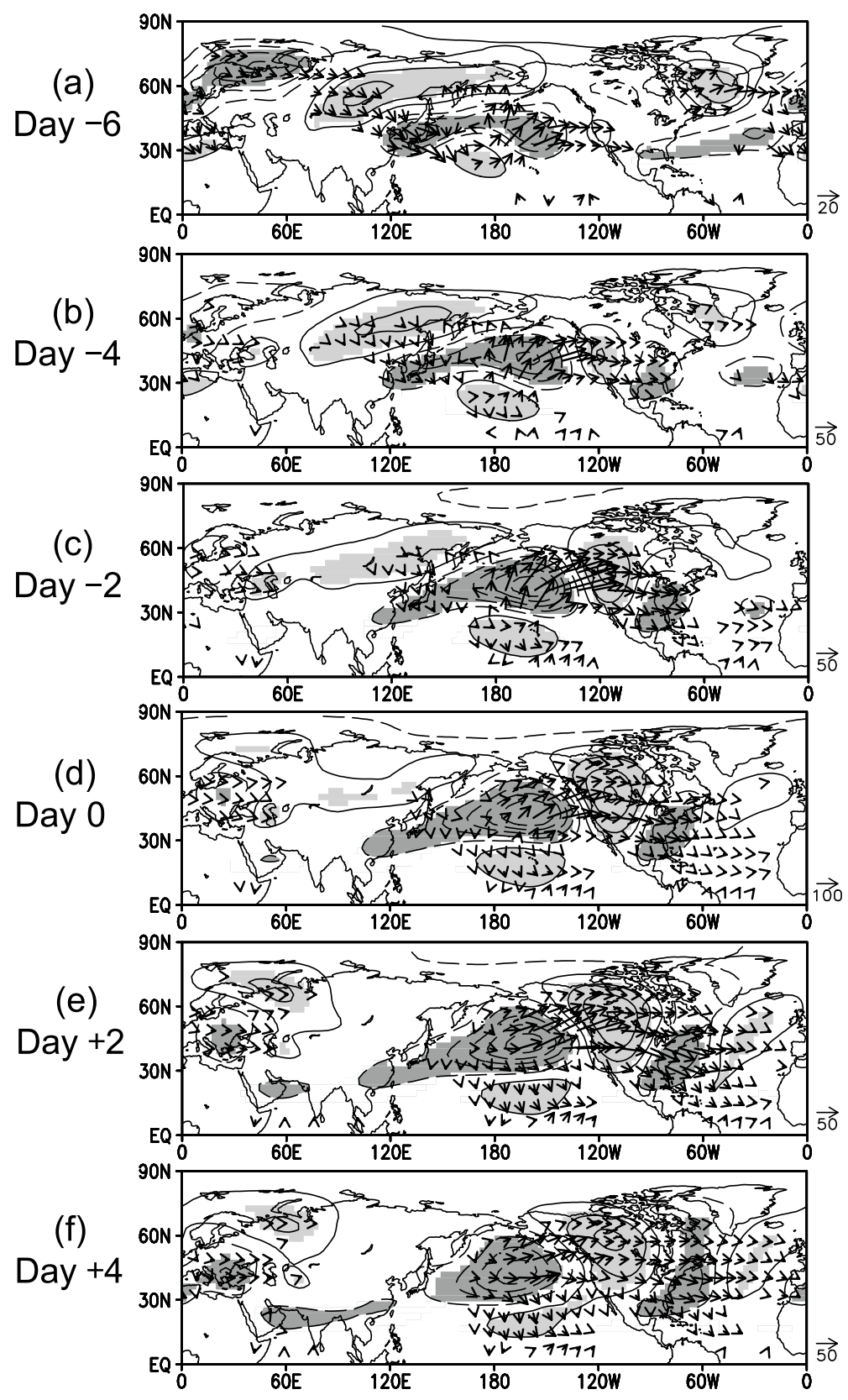

Fig. 3. Lagged composite maps of the anomalous 10-day low-pass filtered $Z 300$ for the positive phase of the PNA. Contour interval is $25 \mathrm{~m}$ in (a) while is $50 \mathrm{~m}$ in (b)-(f). Negative contours are dotted, the zero contour omitted. Shading indicates the anomalies significant at the $95 \%$ level. Arrows represent the wave-activity fluxes (the unit $\mathrm{m}^{2} \mathrm{~s}^{-2}$ ) associated with the composite $Z 300$ anomaly. The scaling for the arrow is shown at the lower right corner.

composite streamfunction tendency fields at each day are projected onto the PNA pattern as defined by the composited low-frequency $\psi 300$ at the peak day for each phase of the PNA. The spatial projection is calculated over $0^{\circ}-70^{\circ} \mathrm{N}, 120^{\circ} \mathrm{E}-60^{\circ} \mathrm{W}$.
The time series of the projection coefficients for the positive and negative phases are shown in Figs. 5 and 6, respectively. Even though we have ignored several terms in Eq. (1) such as the vorticity tilting and eddy viscosity, the sum of all 


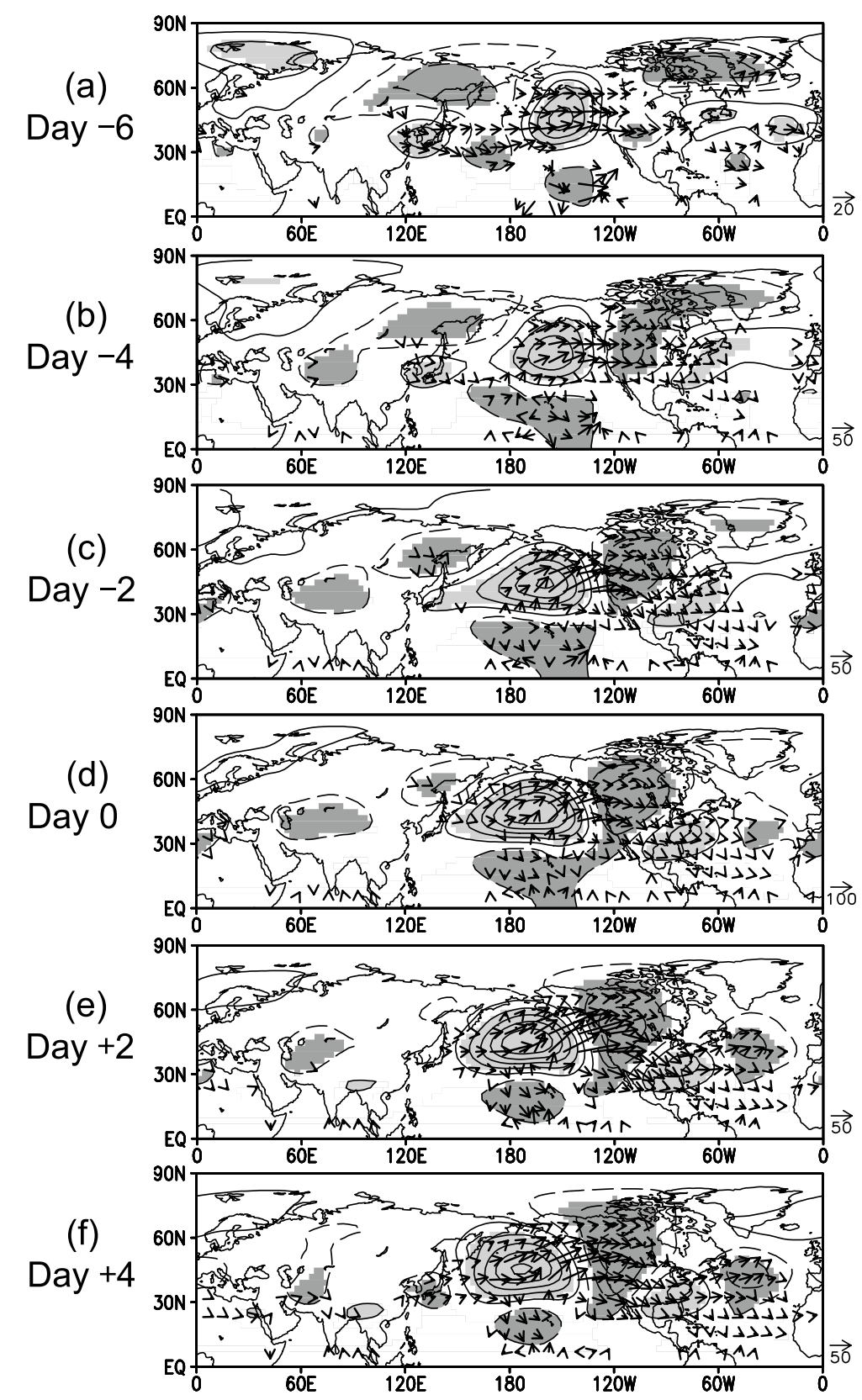

Fig. 4. Same as Fig. 3 but for the negative phase of the PNA.

terms on the right-hand side of Eq. (1), i.e., $\sum_{i=1}^{7} \xi_{i}$ (neglecting the residual) and the left-hand side term $\partial \psi_{L} / \partial t$ are quite similar with each other (Figs. $5 \mathrm{a}$ and $6 \mathrm{a}$ ), indicating that Eq. (1) is appropriate for the PNA-related $\psi_{L}$ in the upper troposphere.

Figures $5 \mathrm{a}$ and $6 \mathrm{a}$ show that the PNA pattern strongly grows at day -4 while decays at about day +5 . The sum of the linear terms, i.e., $\sum_{i=1}^{4} \xi_{i}$, and that of the nonlinear terms, i.e., $\sum_{i=5}^{7} \xi_{i}$, for the positive (negative) phase are shown in Fig. 5b (Fig. 6b). On one hand, for the positive phase, a comparison between linear and nonlinear terms indicates that the growth of the PNA is almost explained by linear processes. Specifically, at day -4 when the tendency of growth is the maximum, the projection of each of the first four $\xi_{i}$ terms (Fig. $5 \mathrm{c}$ ) indicates that the growth of the positive PNA is dominated by $\xi_{2}$ and $\xi_{3}$ while $\xi_{1}$ and $\xi_{4}$ act to 

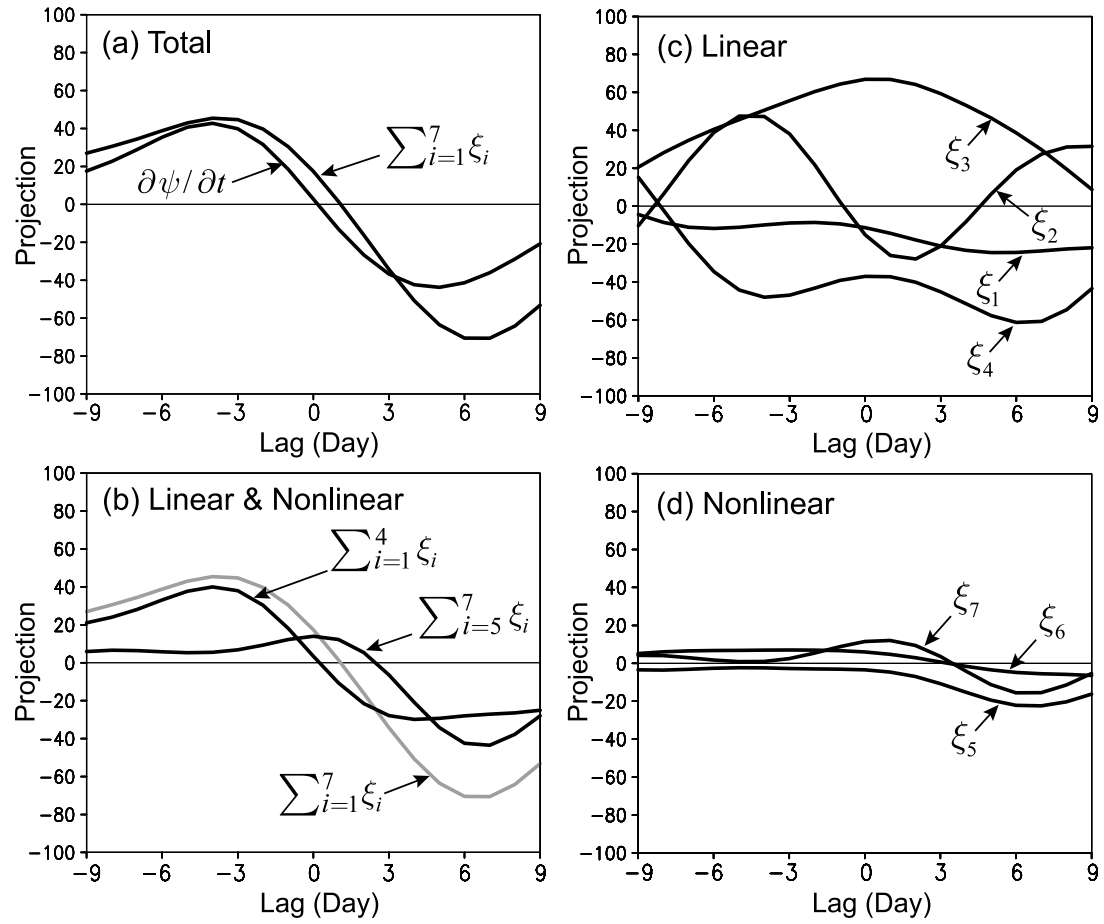

Fig. 5. Time series of the streamfunction tendency patterns projected upon the composite low-frequency $\psi 300$ anomaly at day 0 for the positive phase of the PNA: (a) $\partial \psi / \partial t$ and $\sum_{i=1}^{7} \xi_{i}$, (b) $\sum_{i=1}^{4} \xi_{i}, \sum_{i=5}^{7} \xi_{i}$, and $\sum_{i=1}^{7} \xi_{i}$, (c) $\xi_{1}$ to $\xi_{4}$, and (d) $\xi_{5}$ to $\xi_{7}$. The spatial projection is calculated over $0^{\circ}-70^{\circ} \mathrm{N}, 120^{\circ} \mathrm{E}-60^{\circ} \mathrm{W}$. The units for the ordinate are arbitrary. See text for the definition of each term.
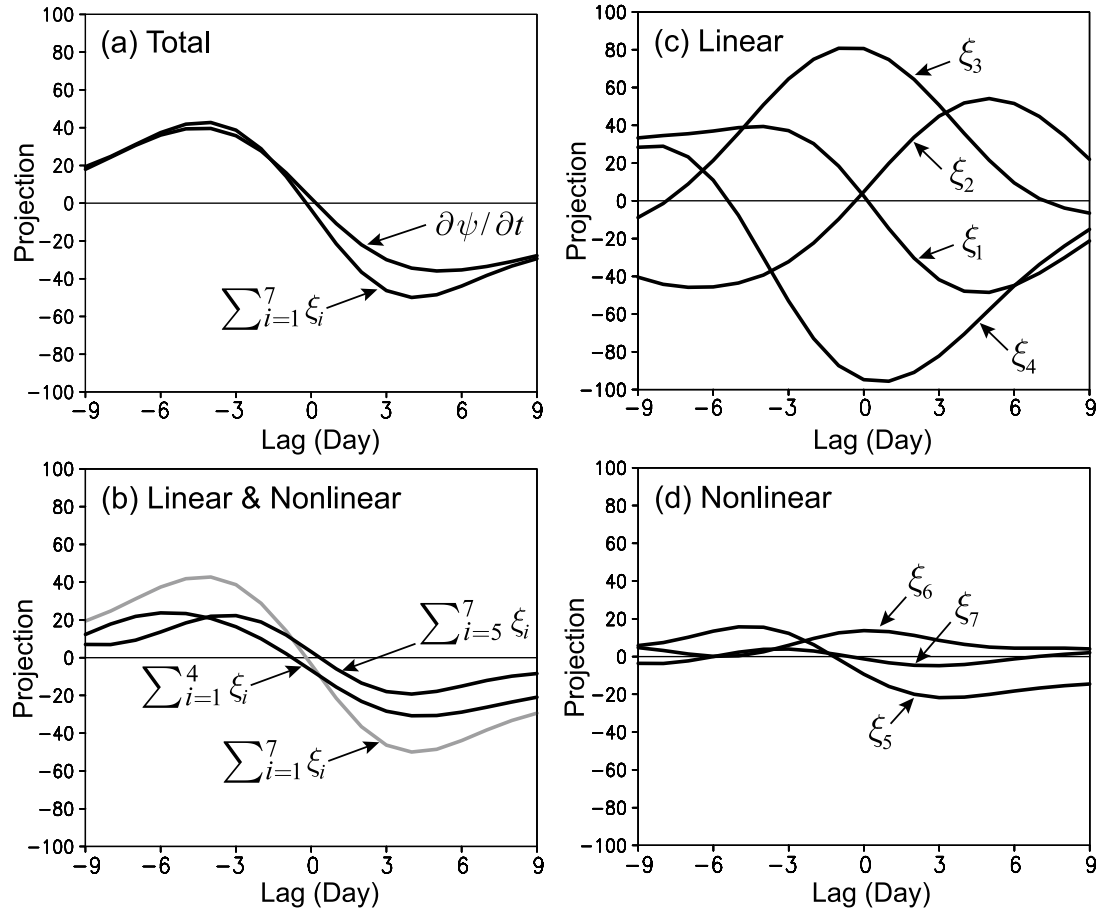

Fig. 6. Same as Fig. 5 but for the negative phase of the PNA. 
(a) Total

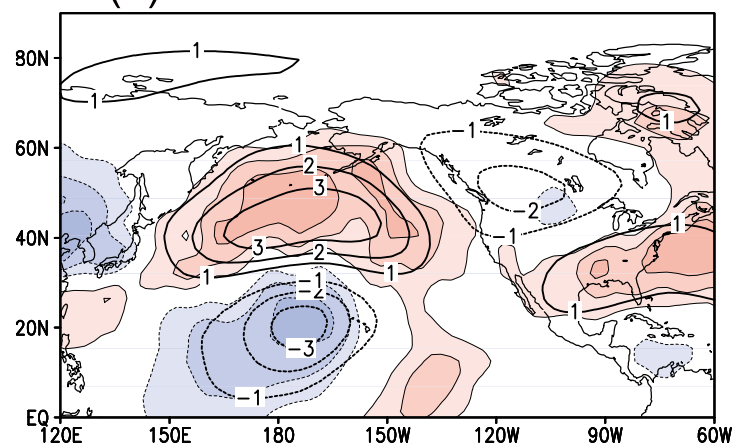

(b) Linear

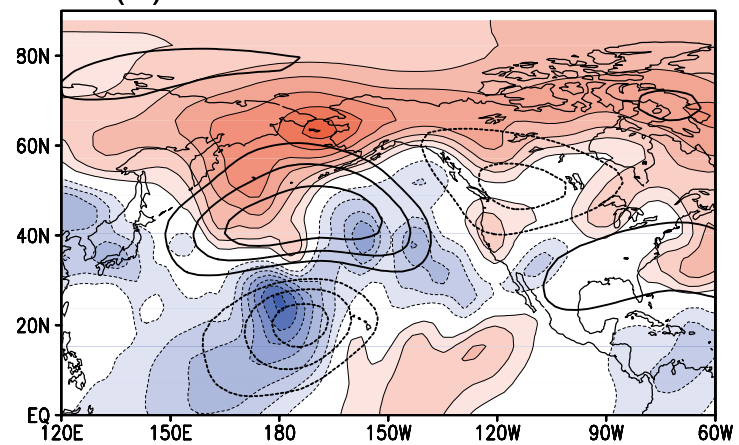

(c) Nonlinear

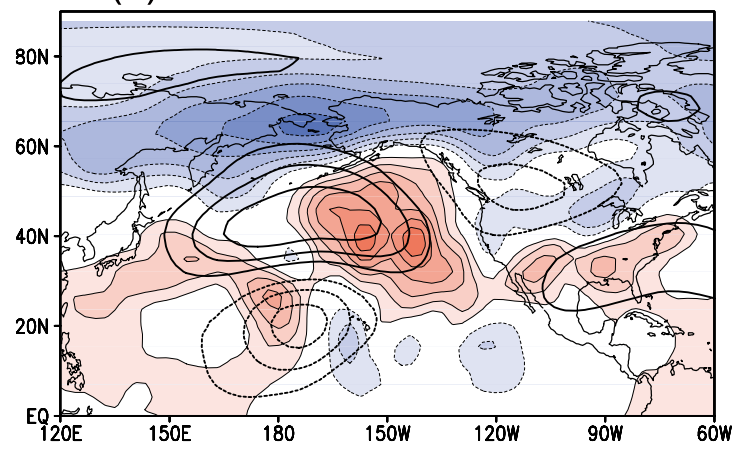

(d) $\xi_{1}+\xi_{2}+\xi_{4}$

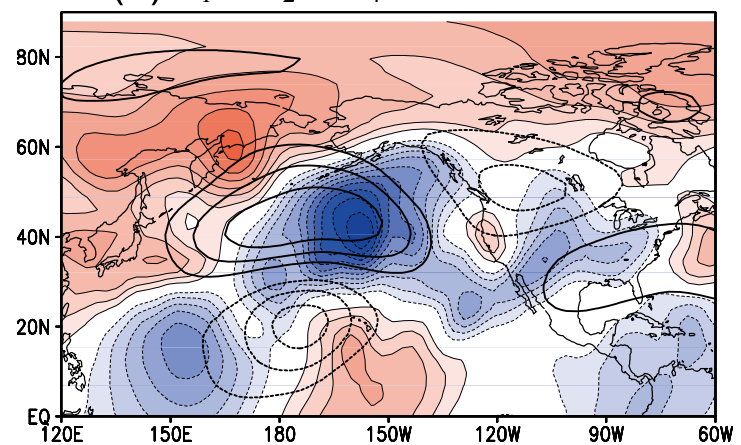

(e) $\xi_{3}$

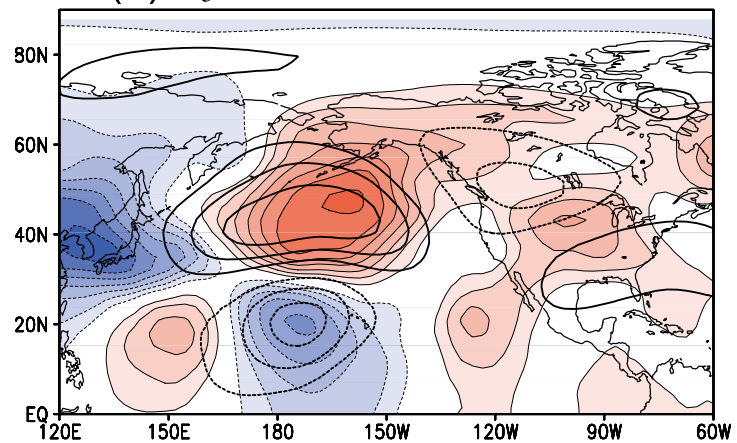

(f) $\xi_{5}$

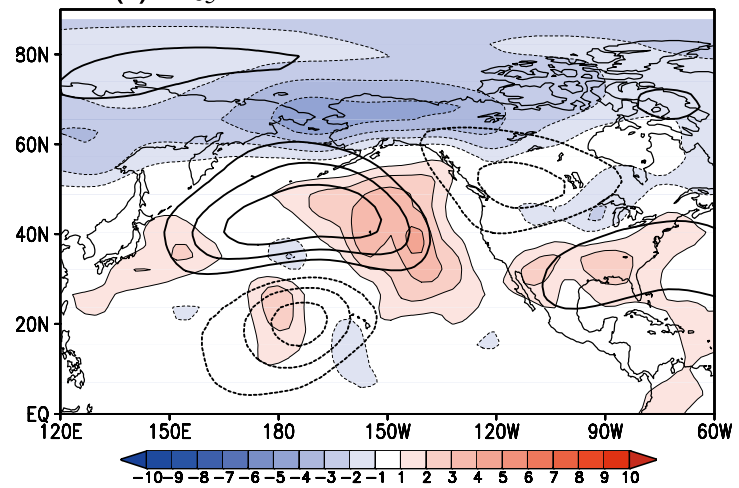

Fig. 7. Composite maps of the streamfunction tendency at day -4 for the negative phase of the PNA: (a) $\sum_{i=1}^{7} \xi_{i}$, (b) $\sum_{i=1}^{4} \xi_{i}$, (c) $\sum_{i=5}^{7} \xi_{i}$, (d) $\xi_{1}+\xi_{2}+\xi_{4}$, (e) $\xi_{3}$, and (f) $\xi_{5}$, which are all indicated by shading (the unit is $10^{6} \mathrm{~m}^{2} \mathrm{~s}^{-1}$ day $\left.{ }^{-1}\right)$. Contours indicate composite map of $\partial \psi / \partial t$ with the interval of $1 \times 10^{6} \mathrm{~m}^{2} \mathrm{~s}^{-1}$ day $^{-1}$. Negative contours are dotted, and zero contour is omitted.

damp. The contributions from every three nonlinear processes are small (Fig. 5d).

On the other hand, for the negative phase at day -4 , the linear and nonlinear terms are found to be comparable in magnitude and are both driving the growth (Fig. 6b). This indicates that the nonlinear processes are not negligible for the growth of the negative PNA. The growth is mainly caused by $\xi_{1}$ and $\xi_{3}$ for linear and by $\xi_{5}$ for nonlinear processes, while $\xi_{2}$ and $\xi_{4}$ work to prohibit the growth (Figs. $6 \mathrm{c}$ and $6 \mathrm{~d})$. The growth mechanism of the PNA is thus dependent on its polarity.

Figure 7 shows the spatial structures of several combinations of tendency terms at day -4 for the negative phase of the PNA superimposed on $\partial \psi_{L} / \partial t$ at that day (contour in Fig. 7). The vorticity budget is mostly balanced except for the North American region (Fig. 7a). When we focus on the 
anticyclonic anomaly over the North Pacific, the linear and nonlinear terms are found to intensify the anomaly over the western and eastern parts, respectively (Figs. $7 \mathrm{~b}$ and $7 \mathrm{c}$ ). Since $\xi_{1}$ and $\xi_{2}$ are largely canceled over the North Pacific region (not shown, but a similar indication is found in Fig. $6 c)^{3}$, their contribution to the net tendency is small and, therefore, the net effect of the linear terms is caused by $\xi_{3}$ and $\xi_{4}$. Indeed, a comparison of the total linear terms (Fig. 7b) with $\xi_{1}+$ $\xi_{2}+\xi_{4}$ (Fig. 7d) and $\xi_{3}$ (Fig. 7e) reveals the linear growth dominated by $\xi_{3}$ while others, especially $\xi_{4}$ (not shown), acting to weaken the anticyclonic anomaly over the North Pacific. Similarly, nonlinear growth is roughly due to $\xi_{5}$ (Figs. 7c and $7 \mathrm{f})$. Although the magnitude of $\xi_{5}$ is much smaller than that of individual linear terms (cf., Figs. 6c and 6d), it can work significantly to amplify the negative PNA over the northeastern Pacific because of the cancellation between linear terms as described.

The spatial patterns of tendency for the positive PNA also show the importance of linear terms, especially $\xi_{3}$ for the growth (not shown). Therefore, it is identified that the primary mechanism for the growth of both phases of the PNA is the barotropic energy conversion from the zonally asymmetric climatological flow $\left(\xi_{3}\right)$. This result is consistent with some previous studies (e.g., Simmons et al. 1983; Branstator 1990, 1992; Kimoto et al. 2001; Feldstein 2002; Watanabe and Jin 2004), In addition, for the negative phase of the PNA, the interaction among low-frequency anomalies $\left(\xi_{5}\right)$ contributes to the growth. The second term in $\xi_{5}$ is of importance (not shown), so that the nonlinearity can be referred to as the nonlinear vorticity advection due to the low-frequency PNA. We leave the details for future studies since it is beyond the scope of this study.

According to the results of the budget analysis, forcing associated with high-frequency transient eddy fluxes $\left(\xi_{6}\right)$ is not crucial for the growth of the PNA, which may not be consistent with some previous studies (e.g., Lau 1988; Nakamura and Wallace 1993; Branstator 1995; Jin et al. 2006a, b). This point will be discussed in more detail in Section 5.2 .

3 This cancellation indicates that the negative PNA does have a character of the barotropic Rossby wave in a zonally uniform flow.

\section{Triggering mechanisms of the PNA}

\subsection{Initial stage of the life cycle}

In this section we further investigate the PNA life cycle, with particular attention to clarify what triggers or initiates the low-frequency PNA. For this purpose, possible precursors of the both phases of the low-frequency PNA pattern are examined. Composite maps of the observed lowfrequency $Z 300$ fields for the positive and negative phases of the PNA at day -9 and associated WAF are shown in Figs. 8a and 9a, respectively. In addition, meridional gradient of the climatological absolute vorticity, $\beta^{*} \equiv \beta-\partial^{2} \bar{u} / \partial y^{2}$, is calculated from composite of $300 \mathrm{hPa}$ climatological zonal wind, $\bar{u}$, for each phase of the PNA at that day (Figs. $8 \mathrm{~b}$ and $9 \mathrm{~b})$. Since this quantity, called effective $\beta$, represents a measure of the waveguide for Rossby wave packets (Hoskins and Ambrizzi 1993), this is used to confirm whether there is a preferred route for the wave energy propagation.

The composite $Z 300$, WAF, and $\beta^{*}$ indicate that the low-frequency anomalies preceding the positive PNA are characterized by two sets of the Rossby wave train; one is originating in the North Atlantic and propagates downstream along a weak waveguide around $60^{\circ} \mathrm{N}$, while another is emanating from the north of the Bay of Bengal (Figs. 8a and $8 \mathrm{~b}$ ). These wave trains meet over Japan and appear to further propagate eastward to the central North Pacific along the Asian jet waveguide.

For the negative phase of the PNA (Figs. 9a and $9 \mathrm{~b}$ ), the low-frequency anomalies at day -9 are characterized by a single Rossby wave train which may be excited over the north of the Bay of Bengal and propagates eastward as in the positive phase. The anomalous height pattern over Eurasia resembles that for the positive phase, but it is not significant and the WAF does not indicate energy propagation from the Atlantic region to the downstream.

One of the notable features observed at day -9 in both phases is that the cyclonic (anticyclonic) anomalies which grow up to be a center of the positive (negative) low-frequency PNA pattern over the central North Pacific are formed as a part of the wave train near the jet exit region (marked by ' $\mathrm{C}$ ' and ' $\mathrm{A}$ ' in Figs. 8a and 9a). As shown in Figs. $5 \mathrm{c}$ and $6 \mathrm{c}$, these anomalies can eventually grow mainly through coupling with the zonal asymmetry of the climatological flow once they are excited. Therefore, delineating an origin 
(a)

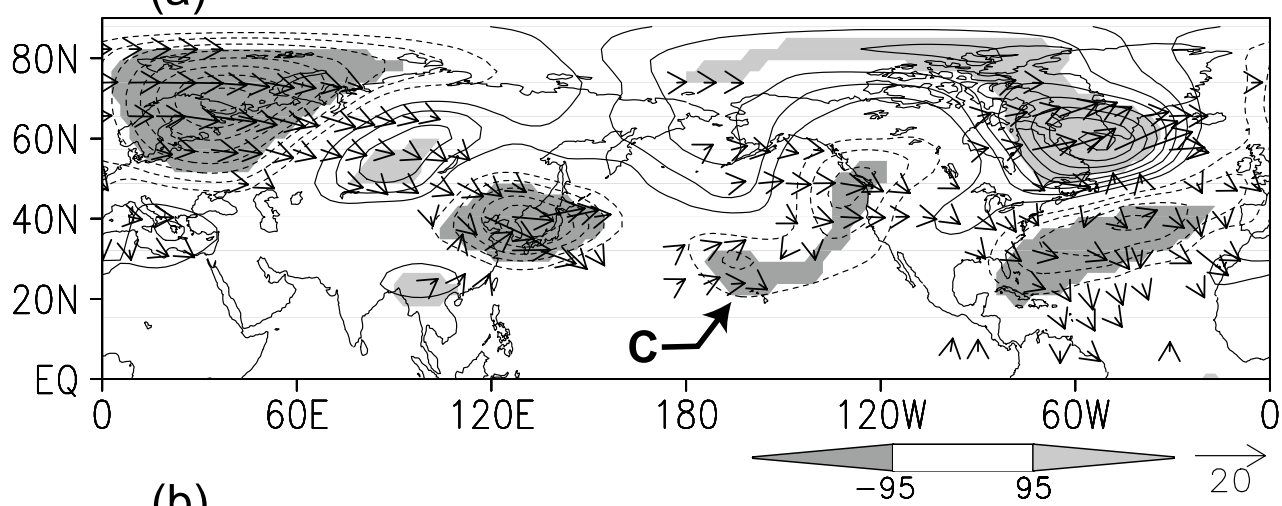

(b)

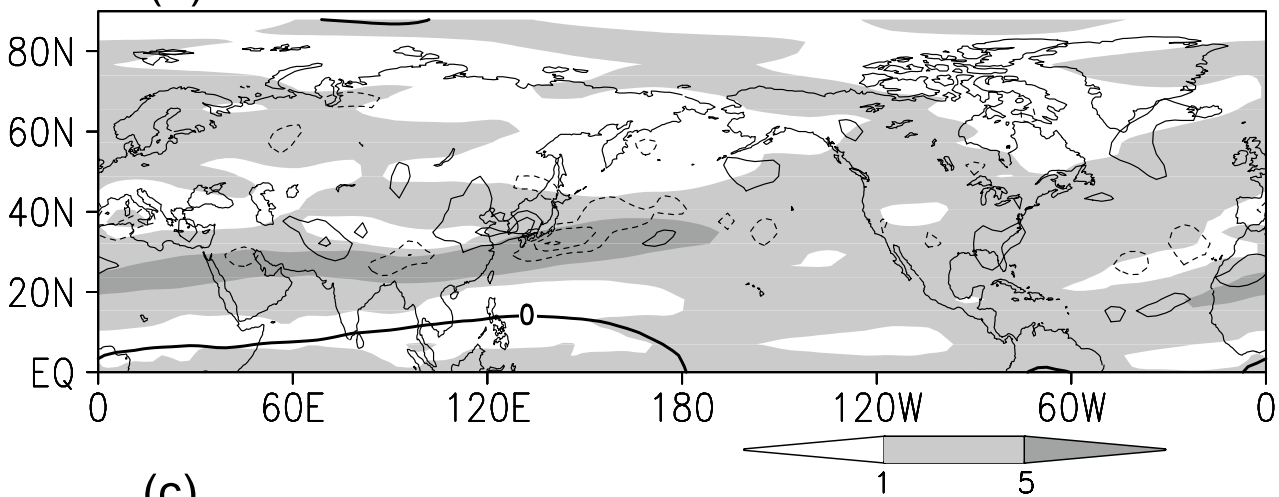

(c)

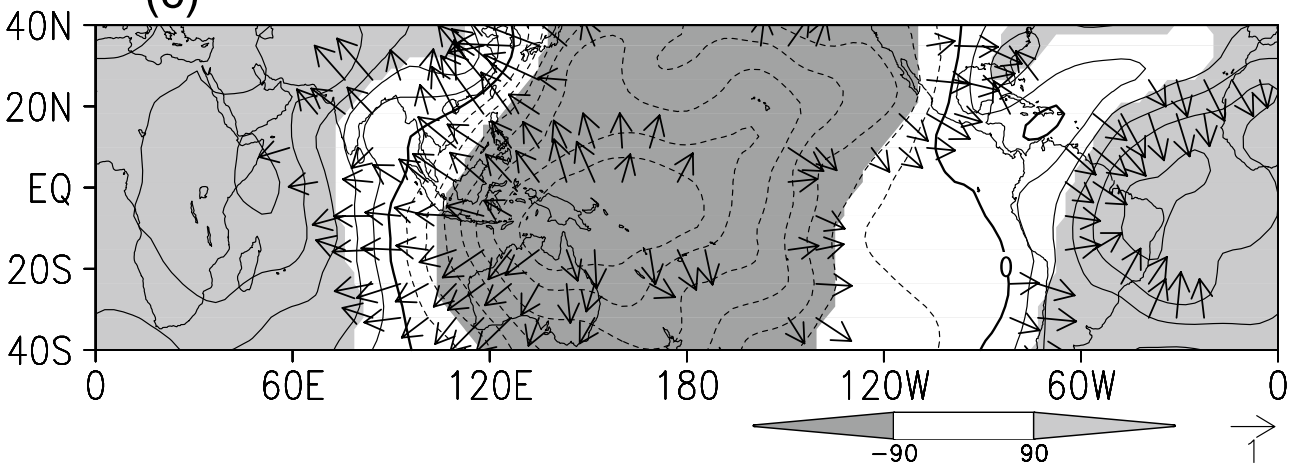

Fig. 8. Composite maps for the positive phase of the PNA at day -9. (a) Low-pass filtered $Z 300$ (contour: interval is $15 \mathrm{~m}$ and the zero contour is omitted, shading: $95 \%$ confidence level), and WAF300 (arrow: $\mathrm{m}^{2}$ $\mathrm{s}^{-2}$ ). (b) $\beta^{*} 300$ of the climatological flow (shade: $10^{-11} \mathrm{~m}^{2} \mathrm{~s}^{-1}$ ), $S_{L} 200$ (contour: interval is $1 \times 10^{-10} \mathrm{~s}^{-2}$ and the zero contour is omitted), and the zero line of $\bar{u} 200$ (thick contour). (c) Low-pass filtered $\chi 200$ (contour: interval is $5 \times 10^{5} \mathrm{~m}^{2} \mathrm{~s}^{-1}$, shading: $90 \%$ confidence level), and $\boldsymbol{V}_{\gamma L} 200$ (arrow: $\mathrm{m} \mathrm{s}^{-1}$, values exceeding 0.4 $\mathrm{m} \mathrm{s}^{-1}$ and significant at the $90 \%$ level are only drawn). Negative contours are dotted, and scaling for the arrow is given at the lower right corner. See text for the explanation of the symbol ' $C$ ' in (a).

of these Rossby wave trains may give us answer to the question of what triggers the low-frequency PNA.

Hereinafter we focus on the Rossby wave train that may originate from the north of the Bay of Bengal as observed in initial stages of the PNA.
In order to identify the origin of these wave trains, Rossby wave sources (Sardeshmukh and Hoskins 1988) are examined. The low-frequency Rossby wave source, $S_{L}$, can be written as 
(a)

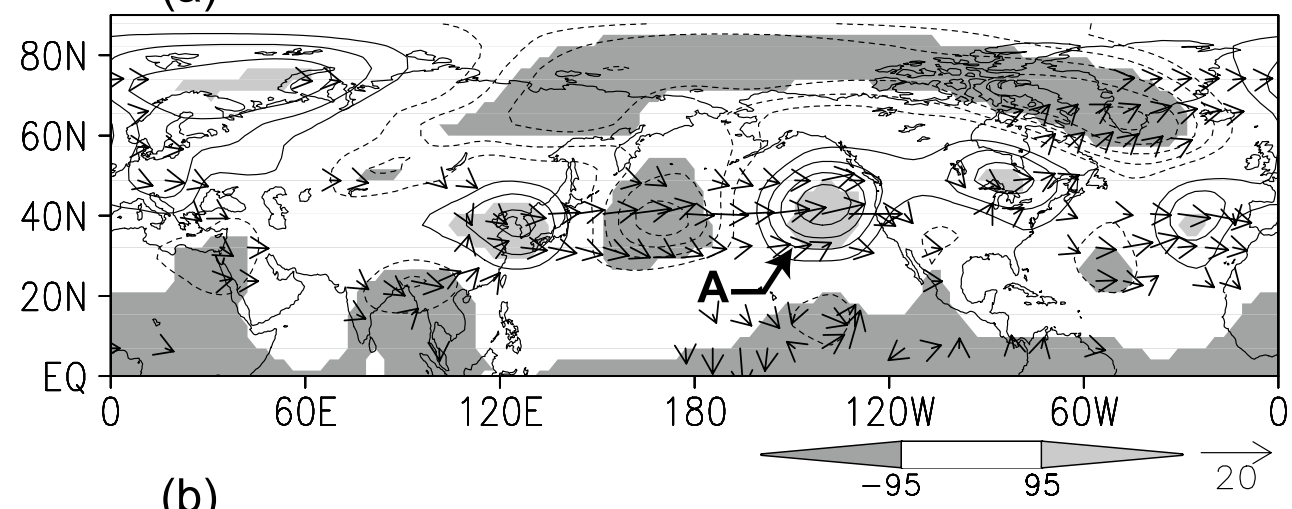

(b)

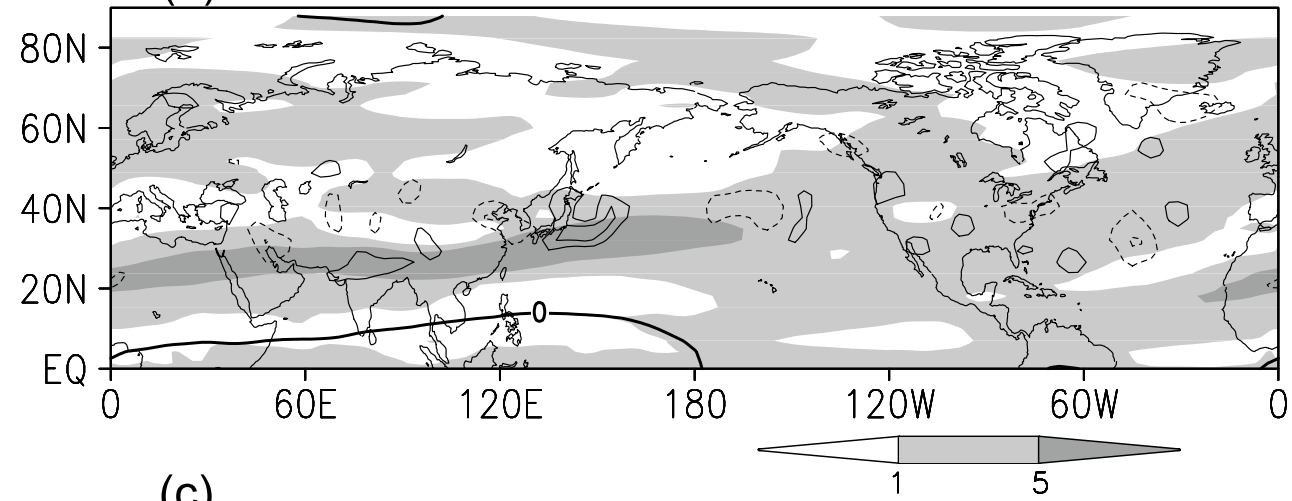

(c)

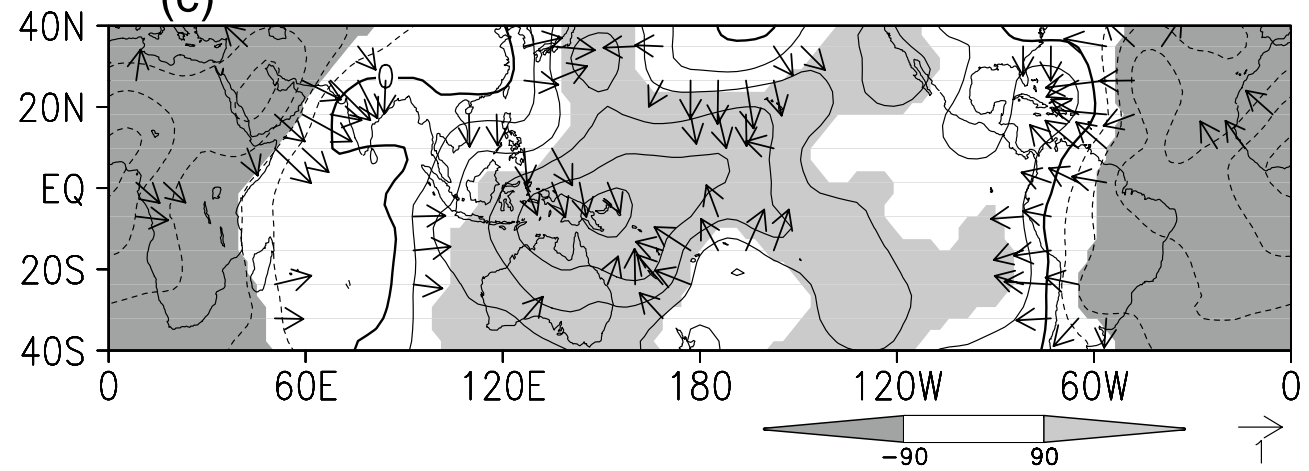

Fig. 9. Same as Fig. 8 but for negative phase of the PNA. See text for the explanation of the symbol 'A' in (a).

$$
\begin{aligned}
S_{L}= & -(\bar{\zeta}+f) D_{L}-\zeta_{L} \bar{D}-\overline{\boldsymbol{V}_{\chi}} \cdot \nabla \zeta_{L} \\
& -\boldsymbol{V}_{\chi L} \cdot \nabla(\bar{\zeta}+f)
\end{aligned}
$$

where the notation of symbols is the same as in Eq. (1) except that $D$ is divergence. Nonlinear terms have been neglected in Eq. (3). Composite maps of the $200 \mathrm{hPa}$ Rossby wave source, $S_{L}$, for each phase of the PNA at day -9 are indicated by contour in Figs. $8 \mathrm{~b}$ and $9 \mathrm{~b}$, respectively. For positive (negative) phase of the PNA, it can be seen that the negative (positive) values of $S_{L}$, which force the anticyclonic (cyclonic) circulation, are observed over the north of the Bay of Bengal where the anticyclonic (cyclonic) height anomaly exists. This coincidence between $S_{L}$ and the height anomaly suggests that the wave train is excited over the north of the Bay of Bengal. This result is consistent with some optimal excitation studies (Borges and Hartmann 1992; Molteni and Palmer 1993) and a study for the sensitivity of the PNA pattern to small perturbations (Corti and Palmer 
(a) positive PNA

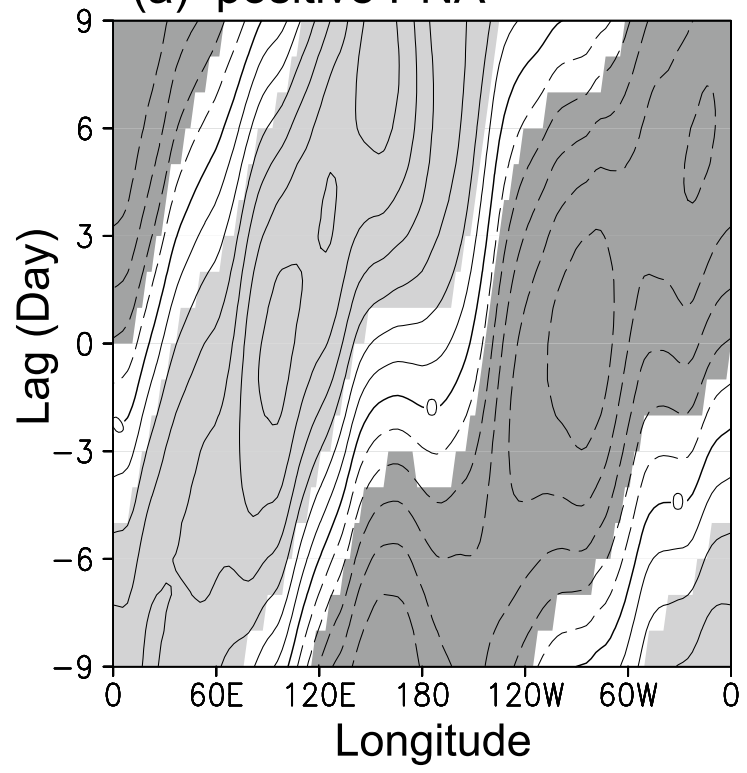

(b) negative PNA

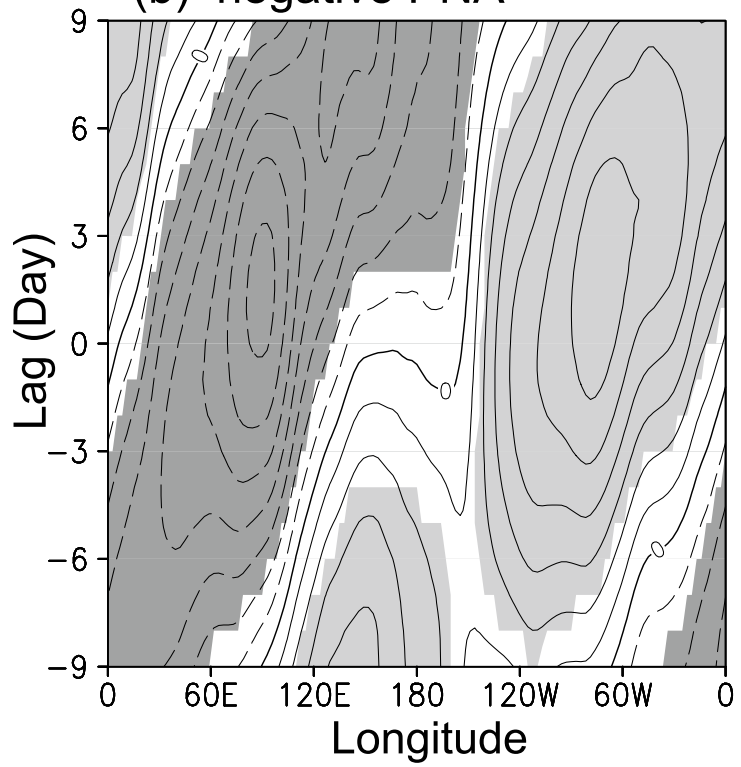

Fig. 10. Hovmöller diagrams for the composite of the PNA for $\tilde{\chi} 200$ along the equator. (a) positive phase,

(b) negative phase of the PNA. Dotted contours indicate negative values and the contour interval is $4 \times$

$10^{5} \mathrm{~m}^{2} \mathrm{~s}^{-1}$. Statistically significant areas exceeding the $95 \%$ confidence level are indicated by shading.

1997).

Sardeshmukh and Hoskins (1988) noted the importance of the vorticity advection by divergent wind associated with tropical convections. Indeed, the last term on the right-hand side of Eq. (3) plays a crucial role for making of $S_{L}$ over that region for both phases of the PNA (not shown). In order to verify possibility that the tropical convective activities can force this wave train upon the Asian jet stream, composite map of $\chi_{L}$ and $V_{x L}$ at $200 \mathrm{hPa}$ for each phase of the PNA at day -9 are shown in Figs. 8c and 9c, respectively. The location and sign of the anomalous divergence are nearly opposite between the positive and negative phases. The anomalies in $\chi_{L}$ are characterized by a zonal wavenumber one structure, which accompanies the divergence (convergence) anomaly over the western equatorial Pacific and the convergence (divergence) anomaly over the equatorial Atlantic and Africa for the positive (negative) phase. Furthermore, the divergent wind anomalies straddle the Asian jet stream over the north of the Bay of Bengal where the climatological absolute vorticity has the largest meridional gradient. These results reinforce that $S_{L}$ over the north of the Bay of Bengal is caused by this divergent wind anomaly in the tropics.
The horizontal structure of $\chi_{L}$ anomalies characterized by the zonal wavenumber one is reminiscent of the MJO. As mentioned in the introduction, several studies suggest that the PNA-like low-frequency variability in the extratropics occurs on intraseasonal timescale in association with the eastward propagation of the MJO. Thus, the Hovmöller diagrams for the composite $\tilde{\chi} 200$ for each phase of the PNA along the equator are examined (Fig. 10). These diagrams show that the life cycle of the PNA accompanies the eastward propagating divergent/convergent signals, which will be projected on the MJO.

To summarize, the results above suggest that the MJO can trigger the low-frequency PNA by exciting a Rossby wave train along the Asian jet waveguide. The Rossby wave train propagating eastward is forced over the north of the Bay of Bengal mainly by the term $-\boldsymbol{V}_{\chi L} \cdot \nabla(\bar{\zeta}+f)$ possibly associated with the MJO. The wave anomaly over the Asian jet exit region will grow up to the PNA by extracting energy from zonally asymmetric climatological flow. A coherent dynamical relationship between the PNA and the MJO will be further examined in the rest of this section. 
(a) EOF 1

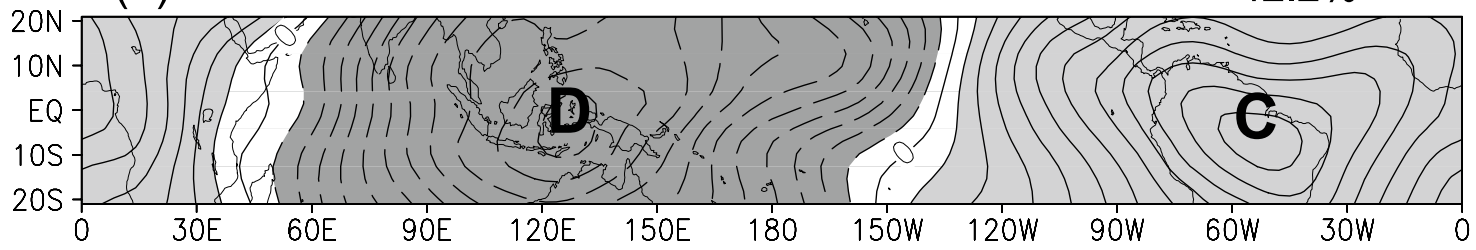

(b) EOF 2

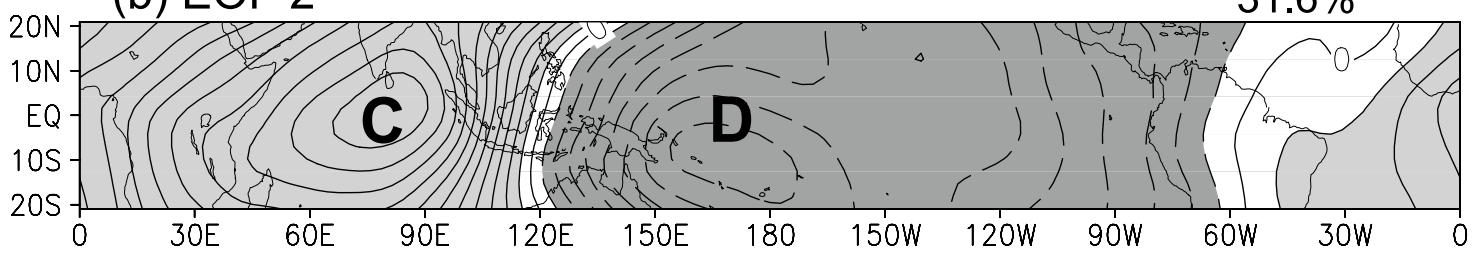

Fig. 11. (a) The first and (b) the second EOF patterns to the daily $\tilde{\chi} 200$ anomalies. Dotted contours denote negative values and the contour interval is $2 \times 10^{5} \mathrm{~m}^{2} \mathrm{~s}^{-1}$. The fractional variance is shown at the upper right corner of each panel. Symbol 'D' and ' $\mathrm{C}$ ' represent the center of divergence and convergence regions, respectively.

\subsection{MJO-PNA coherence}

It is suggested in the preceding subsection that a coherent relationship between the PNA and the MJO exists in association with the PNA life cycle. In an attempt to gain further insight into the PNAMJO relationship from a statistical point of view, we examine multivariate probability density functions (PDFs) of the MJO calculated separately with respect to the polarity of the PNA index.

For this purpose, we first extract the tropical intraseasonal oscillation by applying the EOF analysis to $\tilde{\chi} 200$ for 45 winters over the tropics between $20^{\circ} \mathrm{S}$ and $20^{\circ} \mathrm{N}$. The two leading EOFs (EOF1 and EOF2) account for $42.2 \%$ and $31.6 \%$, respectively, of the total variance on the timescale of 30-60 days. The spatial patterns of these EOFs are shown in Fig. 11, which reveals a set of the wavenumber-one structures. Associated PC time series are oscillatory, in which the PC1 leads the PC2 by quarter of one period (not shown). These two EOFs to $\tilde{\chi} 200$ have been obtained by Lorenc (1984) and represent the eastward propagation of the MJO signal. Now the two-dimensional phase space spanned by the two leading EOFs is defined (the abscissa is EOF1 and the ordinate is EOF2). A trajectory of PCs on this phase plane exhibits one counterclockwise revolution around the origin point per one cycle of the MJO. Since the MJO has a large interannual variability, the trajectories are different in every winters and the distribution of individual samples (45 winters $\times 151$ days) reveals nearly concentric circles (not shown).
The next step is to calculate the two-dimensional PDFs of the MJO using the trajectory plots for the PCs in the phase space. The PDF is computed with a non-parametric density estimation technique called the kernel method (cf., Kimoto and Ghil 1993a). We adopt Epanechnikov kernel as the kernel function, and choose a parameter which controls the smoothness of the density estimation, called the bandwidth $h$, by the least-squares cross validation technique to make a reasonable estimate. In the present analysis, $h$ is estimated as $h=0.42$.

The result calculated using all 6795 samples is shown in Fig. 12a, which reveals nearly a Gaussian-like distribution. In order to examine whether the PDF of the MJO changes depending on the polarity of the low-frequency PNA, the PDFs are calculated separately for the case in which the PNA index is positive or negative (Figs. 12c and 12d). For the positive (negative) case, 1003 (935) samples are used following the criterion that the PNA index exceeds one standard deviation. In these cases, the occurrence probability densities of the MJO exhibit no longer Gaussian-like distribution but rather a significant ${ }^{4}$ lopsided distribution which is roughly anti-symmetric with respect to the sign of the EOF 1 of $\tilde{\chi} 200$. Furthermore, the PDF skewness is also anti-symmetric about the polarity of the PNA. Specifically, for the positive (negative) PNA, the probability density is significantly large (small) in the negative side of the EOF1 whereas small (large) in the positive side. 

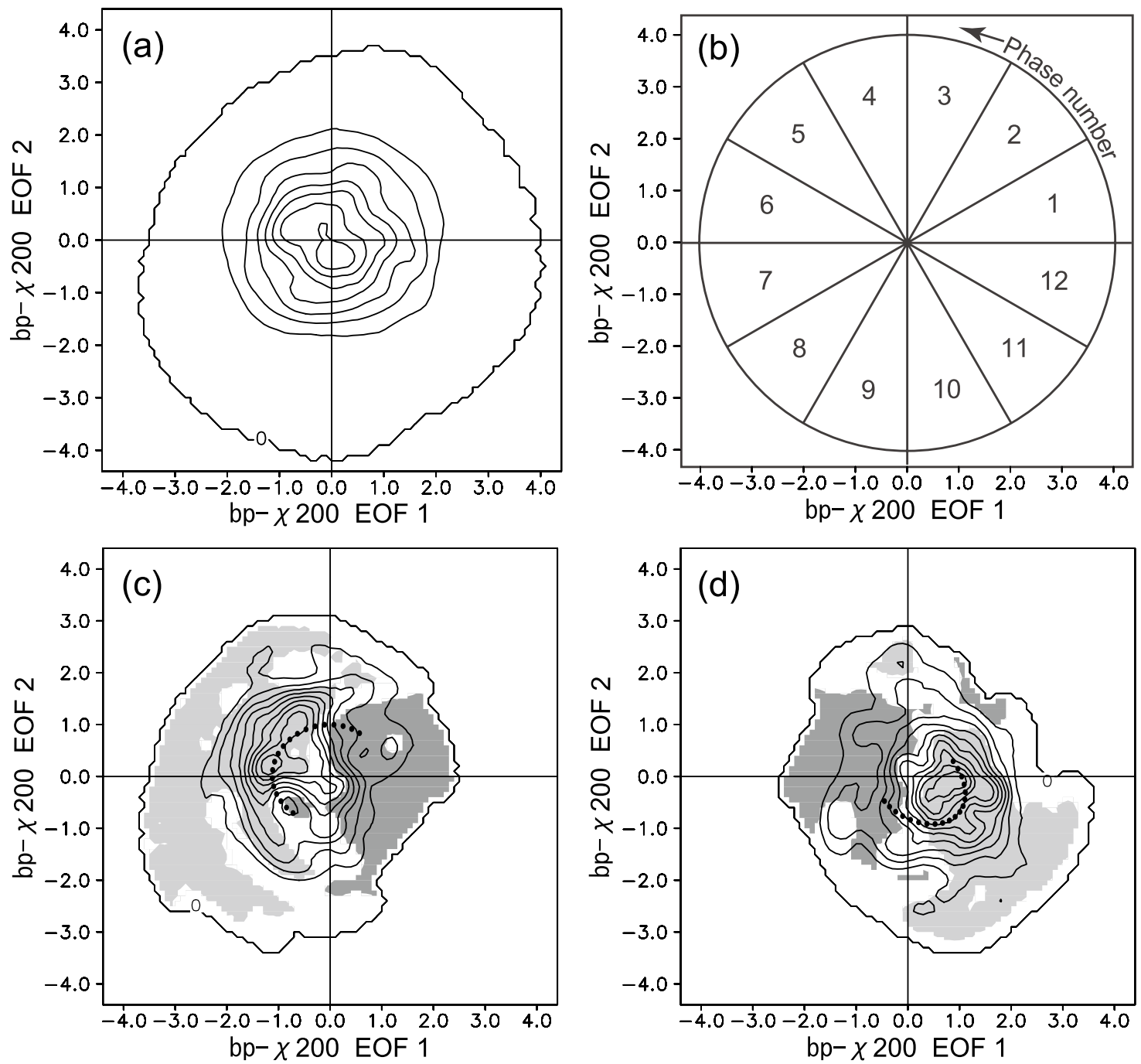

Fig. 12. Two-dimensional PDFs on the phase space spanned by EOF1 and EOF2 of band-pass filtered $\chi 200$. (a) 2D PDF calculated using all 6795 samples. (b) Schematic diagram showing the twelve phases and corresponding phase number. (c) 2D PDF calculated using 1003 samples when the PNA index is positive and exceeded one standard deviation. (d) Same as (c) but using 935 samples when the PNA index is negative. The contour interval is 0.2 . The regions where the probability densities are significantly large or small with the $95 \%$ confidence level are indicated by lightly or heavily shading. See Section 5.2 for the explanation of dots in (c) and (d).

4 The statistical test to the PDF is based on the Monte Carlo method. Namely, one hundred sets of the PNA index which is randomly shuffled in time are first prepared, and then two-dimensional PDFs of the MJO are calculated by using each of the artificial PNA index. Statistical confidence interval is defined at each point of the phase space. The number of random sets having larger or smaller values than the observation determines the statistical significance level.
These features again indicate a systematic coherence between the PNA and the MJO even though the two phenomena have somewhat different timescales. Figures $12 \mathrm{c}$ and $12 \mathrm{~d}$ also indicate the two local maxima roughly along the axis of the EOF1, implying that the positive (negative) low-frequency PNA tends to dominate when the center of anomalous upper-level convergence (divergence) associated with the MJO exists over Indonesia.

Since the anomalous divergence/convergence 
should, in reality, be induced by the anomalies in convective activity, composite for the band-pass filtered OLR anomaly is made with the following procedures. The MJO cycle is divided into 12 phases (per $30^{\circ}$ ) as shown in Fig. 12b, each of which has a serial number. Every sample of the OLR is sorted into these 12 phases using the $\tilde{\chi} 200$ PCs and averaged in each phase to make the composite. In order to distinguish from the composite based on the PNA index, it is hereinafter referred to as the MJO-composite. Figure 13 shows the Hovmöller diagram of the MJO-composite for the band-pass filtered OLR anomaly averaged over the tropics between $10^{\circ} \mathrm{S}$ and $10^{\circ} \mathrm{N}$. It should be noted that the ordinate is not time but phase number illustrated in Fig. 12b. As shown in Fig. 12c, the local maximum of the PDF for the positive PNA resides in phase 6. According to Fig. 13, it is found that the phase 6 corresponds to the period when the positive OLR anomalies associated with MJO exist over the eastern tropical Indian Ocean and the maritime continent.

A concrete picture of the phase-locking between the MJO and the PNA is obtained by looking at the composite maps for the low-pass filtered anomalies as in Section 3.1 but with reference to the MJO. Figure 14a shows the composite low-frequency anomalies in Z300, WAF, and the band-pass filtered OLR anomaly at each phase of the MJO from phases 6 to 12 . The composite procedure is the same as in Fig. 13. As expected, significant $^{5}$ extratropical height pattern which resembles the positive (negative) PNA pattern appears at phase 6 (12). This indicates that the eastward propagation of the MJO accompanies the extratropical low-frequency fluctuations showing a transition between the positive and negative PNAlike anomalies, consistent with previous studies and also with Fig. 12.

While the composite life cycle of the PNA shown in Figs. 2-4 does not capture the phase transition, the MJO composites for the $Z 300$ anomalies in Fig. 14a are more periodic. Nevertheless, the growth and triggering of the PNA-like anomalies are quite similar to those discussed in Sections 3.2 and 4.1. Namely, the positive $Z 300$ anomaly over the central North Pacific at phase

5 The statistical significance level depends on the degree of freedom of sample. For the MJO-composite, when sequential samples belong to one phase, the effective number of freedom is counted one.

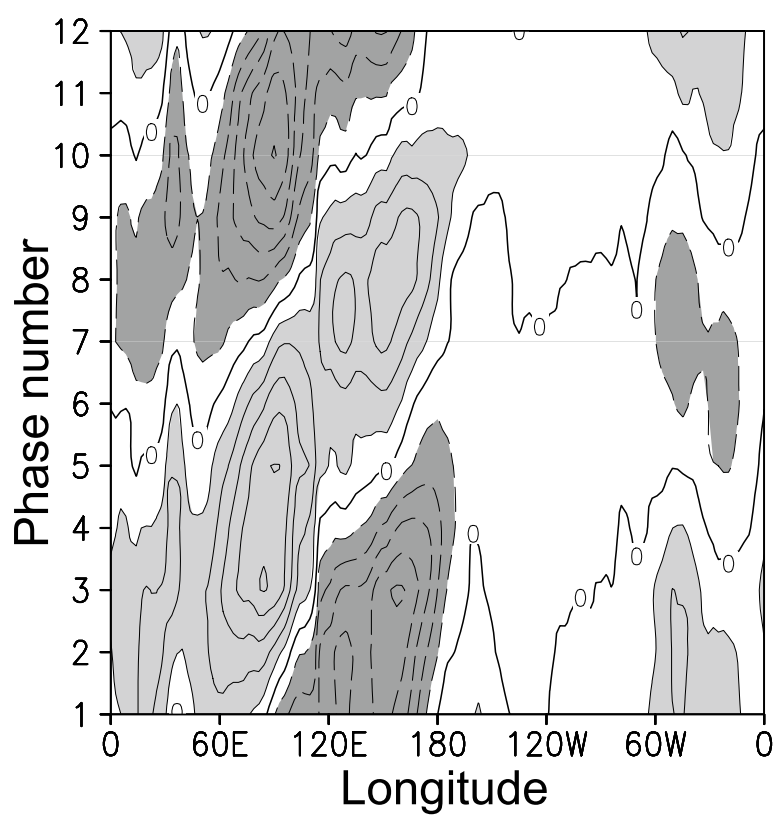

Fig. 13. Hovmöller diagram of the MJO composite for the band-pass filtered OLR anomaly averaged between $10^{\circ} \mathrm{S}$ and 10 ${ }^{\circ} \mathrm{N}$. Dotted contours denote negative values, which represent active convection region. Contour interval is $2 \mathrm{~W} \mathrm{~m}^{-2}$.

12 can be traced back to phase 7 over Japan, when the negative $Z 300$ anomaly is prevailing over the PNA region. At phase 6 , the composite $\mathrm{WAF}$ and OLR suggest a weak negative $Z 300$ anomaly over the north of the Bay of Bengal being forced by anomalous convective cooling around the maritime continent. The composite anomaly in Rossby wave source also reveals that the anomalous divergent wind associated with the MJO plays a crucial role in producing these features (not shown). At phase 7, an anticyclonic anomaly appears over Japan, which may be induced as a part of the Rossby wave train trapped on the Asian jet waveguide. This anticyclonic anomaly gradually moves eastward and develops to the center of the negative PNA-like pattern over the central North Pacific (through phases 8-12). The WAF indicate that these PNA-like patterns are enhanced over the midlatitude, to which the anomalous convective activities over the central tropical Pacific do not contribute. These characteristics for the trigger and growth are consistent with those for the typical low-frequency PNA examined in Section 3.1. In a similar manner, a precursor which eventually 
(a) observation
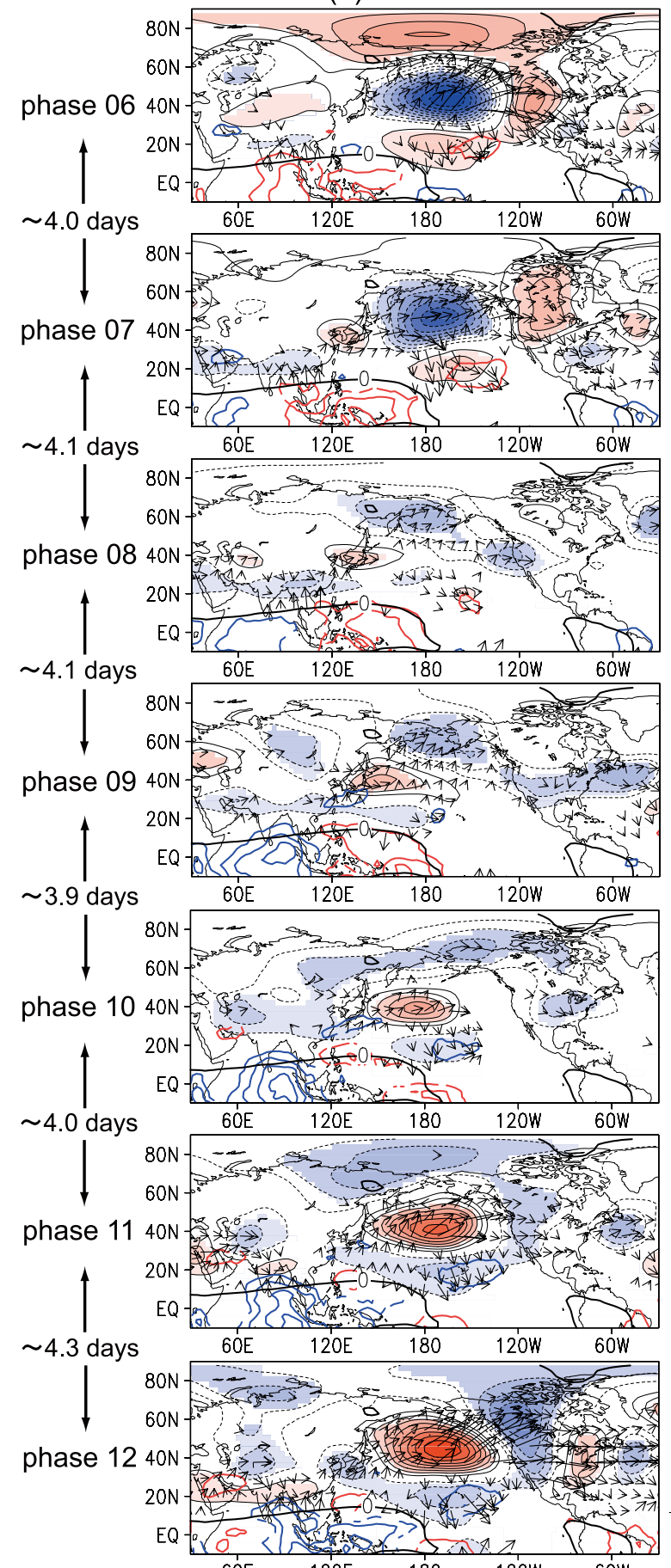

(b) model
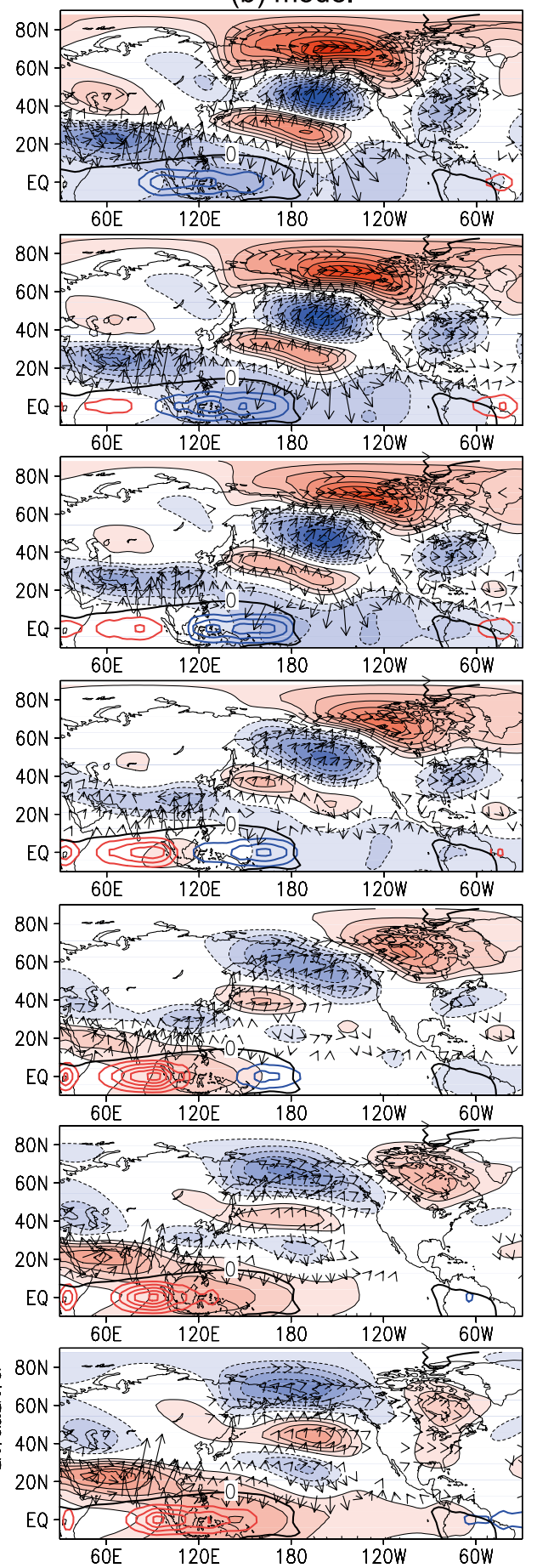

Fig. 14. (a) Observed MJO composites for the low-pass filtered $Z 300$ (contour: interval is $8 \mathrm{~m}$ and the negative contour is dotted without zero contour, shading: $90 \%$ confidence level), WAF300 (arrow: $\mathrm{m}^{2}$ $\mathrm{s}^{-2}$ ), OLR (red and blue contours indicating positive and negative anomalies, which are only drawn where the statistical confidence level exceeds $90 \%$. Contour interval is $8 \mathrm{~W} \mathrm{~m}^{-2}$ ), and the zero line of $\bar{u} 300$ (thick black contour). (b) Same as (a) but for the ensemble average of the forced LBM responses to the propagating equatorial heating. Note that the diabatic heating (cooling) anomaly indicated by the red (blue) contour corresponds to the negative (positive) OLR anomaly in (a). 
develops to the positive PNA-like anomaly can be observed at phase 12 .

The magnitude of the $Z 300$ anomalies is roughly one third of that in the PNA life cycle shown in Figs. 3d and 4d, suggesting that the MJO can explain about $30 \%$ of the low-frequency PNA in terms of its magnitude.

\subsection{Forced LBM responses to propagating heat source}

In order to verify the dynamics between the PNA and the MJO, forced numerical experiments are performed using LBM. The LBM is solved with the time-dependent diabatic heating defined by Eq. (2), which mimics the heat sources associated with the MJO as shown in Fig. 14a. The parameter $\alpha$ which determines the time- and longitudinal-dependent magnitude is estimated from the MJO-composite for the band-pass filtered OLR anomalies averaged between $10^{\circ} \mathrm{S}$ and $10^{\circ} \mathrm{N}$ (Fig. 13). Since the MJO composite is based on phase instead of time, time-dependent composite OLR anomaly is calculated from Fig. 13 by averaging the eastward propagating velocity for each phase as evaluated from the PC trajectories (about 7.3 rad $\left(\mathrm{day}^{-1}\right)$. The eastward propagating forcing is imposed at every model steps using linear interpolation in time. Note that linearity of the model enables us to use an arbitrary absolute magnitude of the forcing.

The extratropical transient responses are dependent on the initial location of the heating, so that a set of 24 integrations each of which is being run for 60 days is performed with the position of initial forcing varying along the equator with intervals of $15^{\circ}$. All the daily outputs of $\chi 200$ after day 16 are projected on the phase space spanned by the EOF1 and 2 of observational $\tilde{\chi} 200$ described in Section 4.2 and then the MJOcomposite is calculated as in Fig. 14a. The slight change in analysis period does not affect the composite results (results not shown). The composite responses in $Z 300$, WAF, and the forcing given to the model are shown in Fig. 14b. A comparison between Figs. 14a and 14b indicates that despite some discrepancies, especially after phase 08 , the LBM can roughly reproduce some observational characteristics such as the triggering, growth and transition of the extratropical anomalies. Similar experiments performed with the zonally uniform basic state show that the extratropical responses are neither PNA-like pattern nor concentrated over the central North Pacific (not shown). This result indicates that the energy conversion from the zonally asymmetrical climatological state plays a crucial role in the formation of the PNA pattern associated with the MJO.

Therefore, the LBM experiments reinforce our finding that the precursor anomaly which eventually grows to the PNA is partly induced by the tropical heating and cooling associated with the MJO and is enhanced over the central North Pacific by extracting energy from the zonally varying climatological state.

The robustness of the responses in Fig. 14b are examined with several additional experiments by changing heating parameters, namely, the vertical profile, latitudinal position, and the eastward propagating velocity. While the responses were somewhat sensitive to the eastward propagating velocity of the heating, they all reproduce the PNA-like structure (not shown). These results indicate that the PNA-like responses strongly depend on the basic state but not on the heating as long as the longitudinal structure, $\alpha(\lambda)$, is prescribed.

The comparison between Figs. 14a and 14b also shows a noteciable difference. While the observed PNA-like anomalies have nearly the same magnitude at phases 6 and 12 (Fig. 14a), the magnitude of the negative PNA-like response at phase 12 is significantly smaller than that of positive one in the LBM (Fig. 14b). Since the model is linear the asymmetric response must be due to asymmetry in heating and cooling, which has been estimated from the observation. Therefore, the observed negative PNA-like anomaly may not be explained solely by the linear process. These results are, suggesting again consistent with the results of the budget analysis in Section 3.2 that nonlinear processes are not negligible for the growth of the negative PNA pattern.

\section{Summary and Discussion}

\subsection{Summary of this study}

In this study, processes governing the life cycle of the low-frequency PNA, especially its trigger and growth, are investigated by means of observational daily composites and associated vorticity budgets.

The results of the vorticity budget analysis illustrate that the positive phase of the PNA tends to grow by linear processes such as the barotropic energy conversion from the zonally asymmetric climatological flow. For the negative phase of the PNA, nonlinear low-frequency vorticity advection 
is as important as the linear processes. Composite life cycle of the PNA shows that at 9 days before the peak of the PNA a pronounced wave train is observed along the Asian jet stream. The wave train which eventually develops to the PNA near the jet exit region appears to be excited over the north of the Bay of Bengal due mainly to anomalous divergent winds associated with the tropical convective activities of the MJO.

A part of the low-frequency PNA variability coherently fluctuates with the MJO even though the two phenomena have somewhat different timescales. PDFs of the MJO calculated separately following the polarity of the PNA index clearly reveal a phase locking between the PNA and the MJO. When the active (inactive) convection associated with the MJO reaches the Bay of Bengal and the western Pacific, occurrence frequency of the negative (positive) phase of the PNA is the highest. The eastward propagation of the MJO accompanies the extratropical low-frequency fluctuations which may help reversing the phase for the lowfrequency PNA. One polarity of the PNA pattern tends to be triggered by the MJO over the north of the Bay of Bengal when another polarity of the PNA is the most energetic. The phase reversal of the PNA-like pattern is well reproduced by the ensemble of the LBM responses forced by the equatorial heating and cooling which mimic the propagating MJO, further supporting the dynamical PNA-MJO connection.

This MJO triggering explains roughly $30 \%$ of the total PNA events, which suggests that, even though the PNA may be inherent to the extratropical atmosphere, a specific tropical forcing is of importance to realize the PNA as dominant mode.

\subsection{Discussion}

In this study, two sets of the lagged composite map are presented. One is based on the PNA index (Figs. 3 and 4) and another is based on the MJO (Fig. 14a). These two composite maps reveal the PNA-MJO coherence from two opposite directions. This is further strengthened by the trajectory of $\tilde{\chi} 200$ composite with reference to the lowfrequency PNA plotted in the MJO phase space (dots in Figs. 12c and 12d; counterclockwise revolution from day -9 to day 9 ). It is evident that the eastward propagating MJO signal is certainly included in $\tilde{\chi} 200$ anomalies associated with the life cycle of the PNA.

The PNA-MJO coupling via the Rossby wave train excited over the north of the Bay of Bengal is consistent with some previous studies examining linkage between the tropics and midlatitude during the MJO life cycle (e.g., Ferranti et al. 1990; Hsu 1996; Higgins and Mo 1997) and optimal excitation studies (e.g., Borges and Hartmann 1992; Molteni and Palmer 1993). Our analysis indicates the importance of vorticity advection by the divergent wind associated with the MJO for the excitation of the wave train. On the other hand, Hsu (1996) suggests the importance of local convergence/divergence over the jet having the large gradient in mean absolute vorticity, that is, the first term on the right-hand-side of Eq. (3). In our analysis, this term was strong over the Japan in the initial stage of the PNA life cycle (not shown, but a similar indication is found in Figs. 8 and 9), which may also have affected the amplification or maintenance of the wave train. Alternatively, Kim et al. (2006) suggest that the importance of vorticity advection by the vertical wind and tilting over that convergence/divergence region where the Asian jet having a strong vertical shear. This effect is not included in the Rossby wave source theory, and is not examined in this study. These discrepancies may imply the complexity of the excitation mechanisms of the wave train associated with the eastward propagating MJO. Furthermore, there is a suggestion that the extratropical circulation anomaly feeds back to the MJO (e.g., Liebmann and Hartmann 1984; Lau and Phillips 1986; Ferranti et al. 1990; Higgins and Mo 1997).

In addition to the forcing in the tropics, there seems to be another precursor triggering the lowfrequency PNA, which was not focused on in the present work. In the initial stage of the positive PNA life cycle (Figs. 3a and 8a), a prominent wave train originates over the North Atlantic and propagates downstream over Eurasia. This wave train quite resembles the Eurasian (EU) teleconnection pattern (Wallace and Gutzler 1981), which indicates the possibility of a preferred transition route from the EU pattern to the PNA. Some studies which examined the extratropical fluctuation associated with the MJO identify a similar pattern (e.g., Lau and Phillips 1986; Knutson and Weickmann 1987; Ferranti et al. 1990).

There may be a drawback in the projection technique in Section 3.2. Although the tendency associated with each term on the right-hand-side of Eq. (1) creates instantaneously the streamfunction 

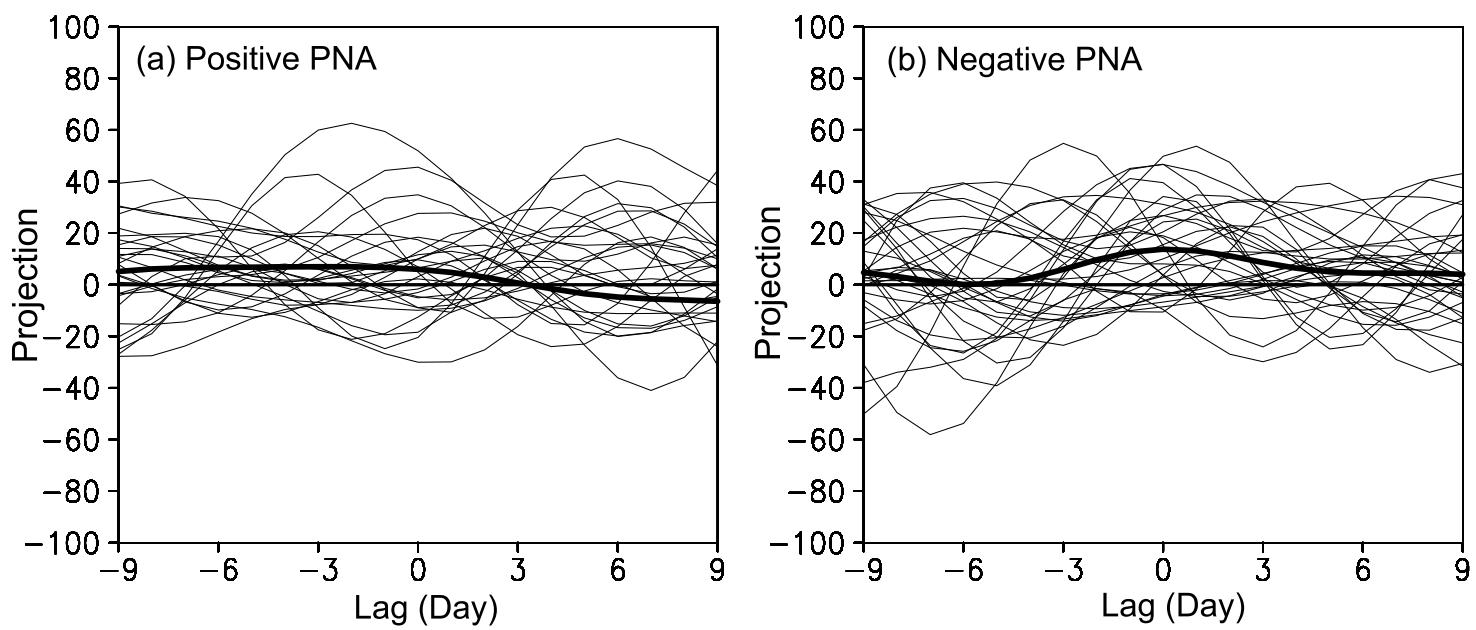

Fig. 15. Same as Fig. 5. but the streamfunction tendency patterns $\xi_{6}$ for each persistent (a) positive, (b) negative PNA event are projected upon the composite low-frequency $\psi 300$ anomaly at day 0 . The average time series is indicated by the thick curve, which correspond to $\xi_{6}$ line in Fig. 5(d) or 6(d).

anomaly, the tendency patterns are projected onto the single PNA pattern at the peak day. Therefore, we should not discuss the role of each term in the initial and final stages when the spatial structure of the tendency is far from the PNA pattern. However, it would be appropriate in the growth and decay stages when the PNA index is sufficiently large or small (see Fig. 2, day -5 to day +5 ).

The results of the budget analysis suggest that feedbacks due to the high-frequency transient eddy fluxes do not play a vital role for the growth of the PNA anomaly, which is inconsistent with previous studies emphasizing the interaction between the low-frequency circulation and storm track. In order to examine the extent to which the high-frequency eddies can force the individual PNA events, time series of the $\xi_{6}$ term in Eq. (1) associated with each persistent PNA event are shown in Fig. 15, following the method described in Section 3.2. Figure 15 indicates that the $\xi_{6}$ term in individual events does affect the PNA anomaly compared to the composite life cycle (thick curve in Fig. 15) and has sufficiently large magnitude compared to the linear term in individual events (not shown). This is largely due to the cancellation of the transient eddy forcing among the events; each $\xi_{6}$ term has a quite different phase at the same day. This feature may not be surprising since the low-frequency PNA accompanies at most several transients during the life cycle because of the short timescale. It is therefore plausible that the individual low-frequency PNA is much affected by nonlinear interaction with the high-frequency eddies through the $\xi_{7}$ term in addition to the highfrequency eddy fluxes, namely, $\xi_{6}$ in the vorticity budget Eq. (1).

Nevertheless, high-frequency transients are systematically modulated during the life cycle of the low-frequency PNA, as illustrated by the composite anomalies in high-frequency eddy kinetic energy at $300 \mathrm{hPa}\left(K_{E} 300\right.$, daily climatology removed) shown in Fig. 16. When it is imposed on the $Z 300$ contours for the low-frequency PNA (including climatology), the eddy energy is obviously weak and zonally elongated for the positive PNA whereas strong and accumulated around the ridge over the central North Pacific for the negative PNA (days 6 to 0 in Fig. 16). The composite $K_{E} 300$ represents the path of high-frequency eddies which can be constrained by the low-frequency flow pattern, but the eddy forcing, i.e., $\xi_{6}$, is due to the tilt in eddy shape, which may be more sensitive to the relative phase between the high- and low-frequency anomalies.

The systematic coherence between the PNA and the MJO demonstrated in this study has important consequences for the general circulation model (GCM) simulation and for predictability of the low-frequency PNA anomaly. Since the MJO triggering appears to explain roughly $30 \%$ of the total PNA event, atmospheric GCM simulations which cannot reproduce the realistic MJO may 
(a) Positive PNA
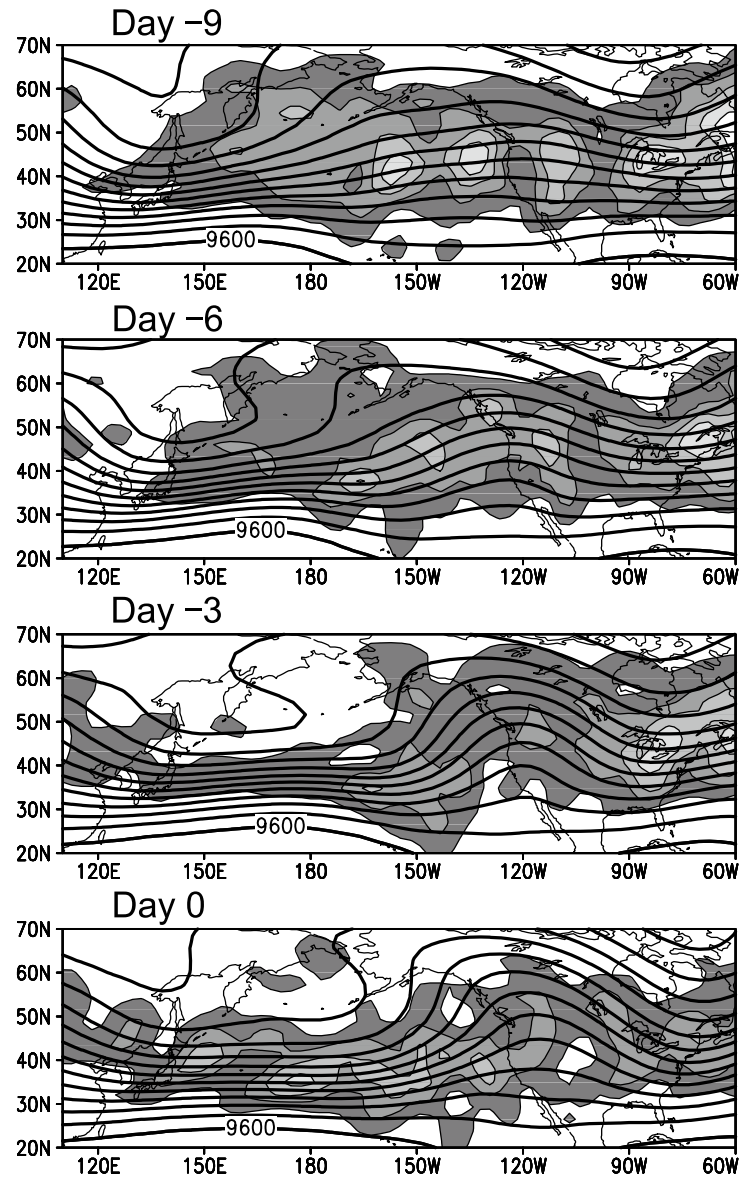

(b) Negative PNA
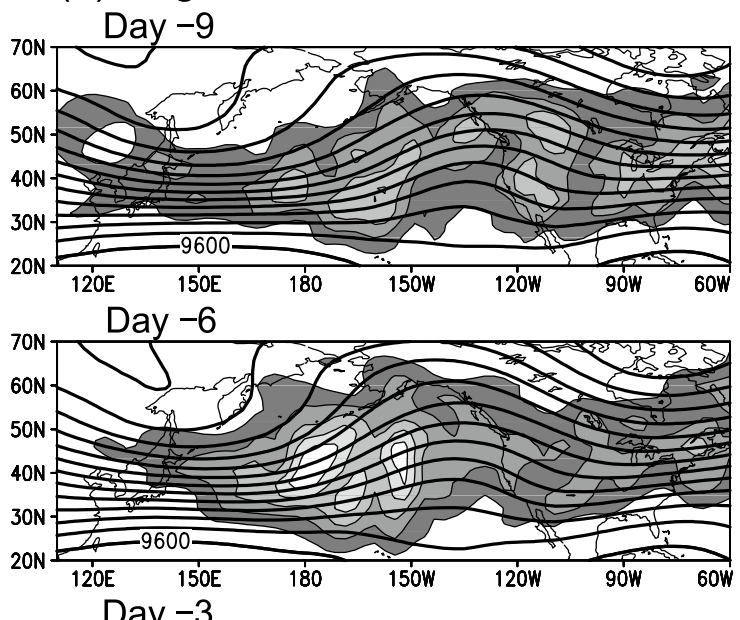
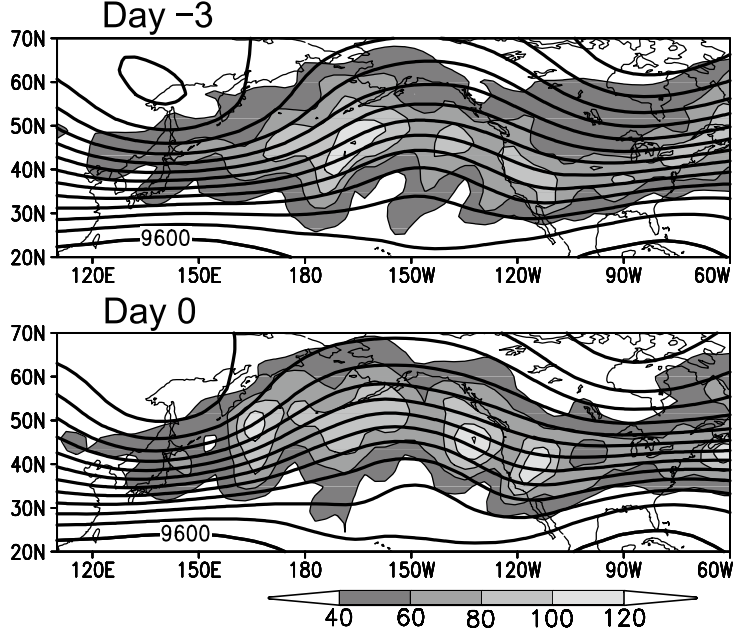

Fig. 16. (a) As in Fig. 3 but of $K_{E} 300$ (shading, $\mathrm{m}^{2} \mathrm{~s}^{-2}$ ) superimposed on the low-frequency $Z 300$ added to daily climatology (contour, interval $100 \mathrm{~m}$ ). (b) As in (a) but for the negative phase of the PNA.

give rise to underestimation the low-frequency PNA variability. Similarly, it is expected that an improvement in predicting the MJO automatically improves extratropical forecast over the PNA region. As discussed above, individual PNA events may be much affected by nonlinear processes more than anticipated from the composite picture, but the MJO-PNA coherence would give us a hope for better PNA forecast which is beyond the predictability limit.

\section{Acknowledgments}

The authors thank M. Kimoto, A. Kubokawa, S. Maeda, H. Mukougawa, H. Nakamura, K. Takaya, and S. Yoden for beneficial comments. Comments by two anonymous reviewers were also helpful. MW is supported by a Grant-in-Aid for Scientific
Research from MEXT, Japan.

\section{References}

Barnston, A.G. and R.E. Livezey, 1987: Classification, seasonality and persistence of low-frequency atmospheric circulation patterns. Mon. Wea. Rev., 115, 1083-1126.

Borges, M. and D. Hartmann, 1992: Barotropic instability and optimal perturbations of observed nonzonal flows. J. Atmos. Sci., 49, 335-354.

Branstator, G., 1990: Low-frequency patterns induced by stationary waves. J. Atmos. Sci., 47, 629-648.

Branstator, G., 1992: The maintenance of low-frequency atmospheric anomalies. J. Atmos. Sci., 49, 1924-1945.

Branstator, G., 1995: Organization of storm track anomalies by recurring low-frequency circula- 
tion anomalies. J. Atmos. Sci., 52, 207-226.

Cai, M. and H.M. van den Dool, 1994: Dynamical decomposition of low-frequency tendencies. $J$. Atmos. Sci., 51, 2086-2100.

Corti, S. and T.N. Palmer, 1997: Sensitivity analysis of atmospheric low-frequency variability. Quart. J. Roy. Meteor. Soc., 123, 2425-2447.

Feldstein, S.B., 2000: The timescale, power spectra, and climate noise properties of teleconnection patterns. J. Climate, 13, 4430-4440.

Feldstein, S.B., 2002: Fundamental mechanisms of the growth and decay of the PNA teleconnection pattern. Quart. J. Roy. Meteor. Soc., 128, 775796.

Ferranti, L., T.N. Palmer, F. Molteni, and E. Klinker, 1990: Tropical-extratropical interaction associated with the 30-60 day oscillation and its impact on medium and extended range prediction. $J$. Atmos. Sci., 47, 2177-2199.

Higgins, R.W. and K.C. Mo, 1997: Persistent north Pacific circulation anomalies and the tropical intraseasonal oscillation. J. Climate, 10, 223-244.

Horel, J.D. and J.M. Wallace, 1981: Planetary-scale atmospheric phenomena associated with the southern oscillation. Mon. Wea. Rev., 109, 813829.

Hoskins, B.J. and T. Ambrizzi, 1993: Rossby wave propagation on a realistic longitudinally varying flow. J. Atmos. Sci., 50, 1661-1671.

Hoskins, B.J. and D.J. Karoly, 1981: The steady linear response of a spherical atmosphere to thermal and orographical forcing. J. Atmos. Sci., 38, 1179 -1196 .

Hsu, H.H., 1996: Global view of the intraseasonal oscillation during northern winter. J. Climate, 9, 2386-2406.

Jin, F.-F., L.-L. Pan, and M. Watanabe, 2006a: Dynamics of synoptic eddy and low-frequency flow feedback. Part I: A linear closure. J. Atmos. Sci., 63, 1677-1694.

Jin, F.-F., L.-L. Pan, and M. Watanabe, 2006b: Dynamics of synoptic eddy and low-frequency flow feedback. Part II: A theory for low-frequency modes. J. Atmos. Sci., 63, 1695-1708.

Karoly, D.J., R.A. Plumb, and M. Ting, 1989: Examples of the horizontal propagation of quasi-stationary waves. J. Atmos. Sci., 46, 2802-2811.

Kim, B.M., G.H. Lim, and K.Y. Kim, 2006: A new look at the midlatitude-MJO teleconnection in the northern hemisphere winter. Quart. J. Roy. Meteor. Soc., 132, 485-503.

Kimoto, M. and M. Ghil, 1993a: Multiple flow regimes in the Northern Hemisphere winter. Part I: Methodology and hemispheric regimes. J. Atmos. Sci., 50, 2625-2643.

Kimoto, M. and M. Ghil, 1993b: Multiple flow regimes in the Northern Hemisphere winter. Part II:
Sectorial regimes and preferred transitions. $J$. Atmos. Sci., 50, 2645-2673.

Kimoto, M., F.-F. Jin, M. Watanabe, and N. Yasutomi, 2001: Zonal-eddy coupling and a neutral mode theory for the Arctic Oscillation. Geophys. Res. Lett., 28, 737-740.

Knutson, T. and K. Weickmann, 1987: 30-60 day atmospheric oscillations: Composite life cycles of convection and circulation anomalies. Mon. Wea. Rev., 115, 1407-1436.

Kushnir, Y. and J.M. Wallace, 1989: Low-frequency variability in the northern hemisphere winter: Geographical distribution, structure, and timescale dependence. J. Atmos. Sci., 46, 3122-3142.

Lau, K.M. and T.J. Phillips, 1986: Coherent fluctuations of extratropical geopotential height and tropical convection in intraseasonal time scales. $J$. Atmos. Sci., 43, 1164-1181.

Lau, N.C., 1988: Variability of the observed midlatitude storm tracks in relation to low-frequency changes in the circulation pattern. J. Atmos. Sci., 45, 2718-2743.

Liebmann, B. and D. Hartmann, 1984: An observational study of tropical-midlatitude interaction on intraseasonal time scales during winter. $J$. Atmos. Soc., 41, 3333-3350.

Lorenc, A.C., 1984: The evolution of planetary-scale $200 \mathrm{mb}$ divergent flow during the FGGE year. Quart. J. Roy. Meteor. Soc., 110, 427-441.

Madden, R.A. and P.R. Julian, 1971: Detection of a 40 -50 day oscillation in the zonal wind in the tropical Pacific. J. Atmos. Sci., 28, 702-708.

Matthews, A., B. Hoskins, and M. Masutani, 2004: The global response to tropical heating in the Madden-Julian oscillation during the northern winter. Quart. J. Roy. Meteor. Soc., 130, 19912011.

Molteni, F. and T.N. Palmer, 1993: Predictability and finite-time instability of the northern winter circulation. Quart. J. Roy. Meteor. Soc., 119, 269298.

Murakami, M., 1979: Large-scale aspects of deep convective activity over the GATE area. Mon. Wea. Rev., 107, 994-1013.

Nakamura, H. and J. Wallace, 1993: Synoptic behavior of baroclinic eddies during the blocking onset. Mon. Wea. Rev., 121, 1892-1903.

North, G., T. Bell, R. Cahalan, and F. Moeng, 1982: Sampling errors in the estimation of empirical orthogonal functions. Mon. Wea. Rev., 110, 699706.

Sardeshmukh, P.D. and B.J. Hoskins, 1988: The generation of global rotational flow by steady idealized tropical divergence. J. Atmos. Sci., 45, 1228 -1251 .

Simmons, A.J., J.M. Wallace, and G. Branstator, 1983: Barotropic wave propagation and instability, and 
atmospheric teleconnection patterns. J. Atmos. Sci., 40, 1363-1392.

Takaya, K. and H. Nakamura, 2001: A formulation of a phase-independent wave-activity flux for stationary and migratory quasigeostrophic eddies on a zonally varying basic flow. J. Atmos. Sci., 58, 608 -627 .

Uppala, S.M. and Coauthors, 2005: The ERA-40 reanalysis. Quart. J. Roy. Meteor. Soc., 131, 29613012.

Wallace, J.M. and D.S. Gutzler, 1981: Teleconnections in the geopotential height field during the
Northern Hemisphere winter. Mon. Wea. Rev., 109, 784-812.

Watanabe, M. and F.-F. Jin, 2004: Dynamical prototype of the Arctic Oscillation as revealed by a neutral singular vector. J. Climate, 17, 2119-2138.

Watanabe, M. and M. Kimoto, 2000: Atmosphereocean thermal coupling in the North Atlantic: A positive feedback. Quart. J. Roy. Meteor. Soc., 126, 3343-3369.

Watanabe, M. and M. Kimoto, 2001: Corrigendum. Quart. J. Roy. Meteor. Soc., 127, 733-734. 